\title{
Das Karlsruher Lissabon-Urteil: Bedingungen, Grenzen, Orakel und integrative Optionen
}

\author{
Peter-Christian Müller-Graff*
}

Das Urteil des Bundesverfassungsgerichts (BVG) vom 30. Juni $2009^{1}$ zur Frage der Vereinbarkeit des Zustimmungsgesetzes zum Vertrag von Lissabon ${ }^{2}(\mathrm{VvL})$ und des diesbezüglichen Grundgesetzänderungsgesetzes ${ }^{3}$ mit dem Grundgesetz (GG) enthält das erwartete Ergebnis. Zustimmungs- und Änderungsgesetz sind verfassungsgemäß, freilich ,nach Maßgabe der Gründe“. ${ }^{4}$ Dieses einstimmige Votum des Zweiten Senats bestätigt das Urteil derjenigen, die dem $\mathrm{VvL}$ (wie schon den dem $\mathrm{VvL}$ im Wesentlichen zugrunde liegenden Neuerungen des Verfassungsvertrages (VVE) ${ }^{5}$ ) eine systemrationale Entfaltung des bisherigen primärrechtlichen Integrationsmodells ohne revolutionäre Elemente bescheinigt hatten. ${ }^{6}$ $\mathrm{Zu}$ einem Widerspruch des VvL zum GG hätte es daher nur kommen können, wenn das GG seine klare Staatszielausrichtung auf die europäische Integration in seiner Präambel vom 23. Mai $1949^{7}$ zwischenzeitlich fundamental geändert hätte. Der Text des GG ist jedoch in dieser Hinsicht seit sechzig Jahren unverändert und wurde im Jahre 1992 durch die Einfügung des Artikels über die Europäische Union (Art. 23 Abs. 1 GG) bekräftigt und konkretisiert. ${ }^{8}$ Zugleich beanstandet das Urteil aber das projektierte Begleitgesetz zur Ausweitung und Stärkung der Rechte des Bundestages und Bundesrates in Angelegenheiten der Europäischen Union ${ }^{9}$ als verfassungsrechtlich in definiertem Umfang nicht hinreichend ${ }^{10}$ und verbietet deshalb (rechtlich nicht zweifelsfrei ${ }^{11}$ ) bis zur verfassungsgemäßen Ausgestaltung dieser Rechte die Hinterlegung der Ratifikationsurkunde. ${ }^{12}$

* Prof. Dr. Dres. h.c. Peter-Christian Müller-Graff, Universität Heidelberg.

1 Verbundene Rechtssachen 2 BvE 2/08, 2 BvE 5/08, 2 BvR 1010/08, 2 BvR 1022/08, 2 BvR 1259/08, 2 BvR $182 / 09$.

2 Gesetz zum Vertrag von Lissabon vom 13. Dezember 2007 vom 08.10.2008, BGB1. 2008 II S. 1038.

3 Gesetz zur Änderung des Grundgesetzes (Artikel 23, 45 und 93) vom 08.10.2008, BGB1. 2008 I S. 1926.

4 Vgl. Rdz. 207 des Urteils. Bindungsgegenstand und Bindungsfolgen von Urteilen des BVG sind indes nicht immer klar abzuschätzen, vgl. zu diesem Problem Hans Lechner/Rüdiger Zuck: Bundesverfassungsgerichtsgesetz. Kommentar, 5. Aufl., München 2006, § 31 Rdz. 30ff., 33ff.

5 Vgl. dazu Peter-Christian Müller-Graff: Strukturmerkmale des neuen Verfassungsvertrages für Europa im Entwicklungsgang des Primärrechts, in: integration 3/2004, S. 186-201.

6 Vgl. dazu z.B. Peter-Christian Müller-Graff: Der Vertrag von Lissabon auf der Systemspur des Europäischen Primärrechts, in: integration 2/2008, S. 123-144.

7 Zur europäischen Integration als Staatszielbestimmung in der Präambel vgl. statt vieler z.B. Peter M. Huber, in: Michael Sachs (Hrsg.): Grundgesetz. Kommentar, 3. Aufl., München 2003, Präambel, Rdz. 41. Sie gilt als Ausdruck „offener Staatlichkeit“, vgl. Klaus Vogel: Die Verfassungsentscheidung des Grundgesetzes für eine internationale Zusammenarbeit. Ein Diskussionsbeitrag zu einer Frage der Staatstheorie sowie des geltenden deutschen Staatsrechts, Tübingen 1964, S. 42ff.

8 Gesetz zur Änderung des Grundgesetzes vom 21.12.1992, BGB1. 1992 I S. 2086; dazu statt vieler z.B. Rudolf Streinz, in: Michael Sachs (Hrsg.): Grundgesetz. Kommentar, 3. Aufl., München 2003, Art. 23 Rdz. 1ff.; Carl Otto Lenz: Ausbrechender Rechtsakt, in: Frankfurter Allgemeine Zeitung, 08.08.2009.

9 Entwurf eines Gesetzes über die Ausweitung und Stärkung der Rechte des Bundestages und des Bundesrates in Angelegenheiten der Europäischen Union, in: Bundestagsdrucksache 16/8489 vom 11.03.2008.

10 Vgl. namentlich Rdz. 406-419 des Urteils.

11 Gegen die rechtliche Zulässigkeit dieses Hinterlegungsverbots aus dem Gesichtspunkt der Verletzung des Verhältnismäßigkeitsgrundsatzes Lenz: Ausbrechender Rechtsakt, 2009.

12 Vgl. Urteilstenor 4b. 
Maßgeblich für die verfassungsrechtliche Beurteilung eines Zustimmungsgesetzes zu neuen primärrechtlichen Integrationsschritten ist zuallererst der Text des GG, auch wenn er im Streitfall der streitentscheidenden Sprechhilfe des dazu authentisch berufenen Interpreten bedarf. Ob dazu im vorliegenden Falle eine (mit sieben zu eins getragene) sehr weit ausgreifende Begründung im Umfang von teils redundanten 421 Einzelschritten erforderlich war, ${ }^{13}$ kann als Frage judikativer Stilpräferenz dahinstehen. Immerhin betrifft sie einen umfänglichen Integrationsvertrag. Sie ist in sich übersichtlich tiefengegliedert, wissenschaftlich niveaureich angelegt und spezifisch konzeptionsgetragen entwickelt, nämlich aus dem konstitutiven Prinzip personaler Freiheit, das sich im demokratischen Teilhabeanspruch der Bürger in Gestalt des Wahlrechts zum Bundestag verwirklicht. ${ }^{14}$ Sie wird indes vor allem ${ }^{15}$ wegen der daraus abgeleiteten weitgreifenden, im Vergleich zum Maastricht-Urteil ${ }^{16}$ schärfer artikulierten rechtlichen, politischen und judikativen Integrationsbedingungen und Schranken wie auch wegen des Gebrauchs vieler hochabstrakter wertungsoffener Begrifflichkeiten, die der Text des GG nicht kennt, zugleich Quell zahlreicher rechtlicher und integrationspolitischer Fragen (und einer vorhersehbaren Flut von Abhandlungen) werden. Darauf deuten bereits jetzt die ersten Reaktionen. Denn thematisch verschränkt das Urteil die verfassungsrechtliche Dimension insbesondere des Demokratie- und Souveränitätsprinzips mit der europapolitischen und europarechtlichen Dimension und betrifft darin zugleich auch zentral die Frage der im Urteil vorgenommenen Selbstaufwertung der Rolle des BVG im System der innerstaatlichen und europäischen Gewaltenteilung. Während allererste journalistische Stimmen in der Begründung noch einen „Weckruf“17 oder sogar eine „Sternstunde" 18 sahen, erkennt eine Phalanx kritischer oder besorgter Stimmen unter anderem eine einseitig restriktive Auslegung des integrationsoffenen Grundgesetzes,${ }^{19}$ ein ,sehr weites“ Hervorwagen Karlsruhes, ${ }^{20}$ eine ,Totalaufsicht des Verfassungsgerichts“ ${ }^{21}$ eine „Entmündigung der Politik“, 22 die Etablierung einer ,,justizgeprägten Demokratie“, 23 ein politisches Manifest der Richter, ${ }^{24}$ eine „Blockade der Europapolitik Deutschlands“ ${ }^{25}$ „,Deutsche Inte-

13 Skeptisch zur Länge etwa Christian Calliess: Unter Karlsruher Totalaufsicht, in: Frankfurter Allgemeine Zeitung, 27.08.2009; Christoph Schönberger: Lisbon in Karlsruhe: Maastricht's Epigones At Sea, in: German Law Journal 8/2009, S. 1201-1218, hier S. 1201-1202; Daniel Halberstam/Christoph Möllers: The German Constitutional Court says "Ja zu Deutschland", in: German Law Journal 8/2009, S. 1241-1257, hier S. 1256-1257.

14 Weichenstellung in Rdz. 210, 212 des Urteils: Ausgangspunkt des GG beim ,Eigenwert und der Würde des zu Freiheit befähigten Menschen“.

15 Auffällig ist aber auch schon am Ansatz die Ausblendung föderativer Eigenheiten in der Legitimationsfrage erkennbar bereits auf deutscher Ebene in Gestalt des Bundesrates - (vgl. zu diesem Problem namentlich Schönberger: Lisbon in Karlsruhe, 2009) und die gedanklich staatskollektive Einhegung der Freiheit.

16 BVerfGE 89, 155.

17 So Reinhard Müller: Weckruf aus Karlsruhe, in: Frankfurter Allgemeine Zeitung, 01.07.2009.

18 So Heribert Prantl: Europäische Sternstunde, in: Süddeutsche Zeitung, 01.07.2009.

19 So Lenz: Ausbrechender Rechtsakt, 2009. Das Urteil des Bundesverfassungsgerichts zum Vertrag von Lissabon legt das integrationsoffene Grundgesetz in einer einseitig restriktiven Weise aus. Das Gericht begibt sich in eine politische Rolle und behindert die Schaffung eines vereinten Europa. Kritisch auch Roland Koch: Wir brauchen mehr Europa, in: Welt am Sonntag, 09.08.2009 und Jürgen Rüttgers, vgl. Frankfurter Allgemeine Zeitung: Kritik an Karlsruhe auch von Rüttgers, 10.08.2009.

20 So Norbert Röttgen im Interview: „Karlsruhe hat sich sehr weit hervorgewagt“. Vgl. Wulf Schmiese/Reinhard Müller: CDU kritisiert Vorschläge der CSU, in: Frankfurter Allgemeine Zeitung, 10.08.2009.

21 Calliess: Unter Karlsruher Totalaufsicht, 2009; dagegen Christian Hillgruber: Die besseren Europäer, in: Frankfurter Allgemeine Zeitung, 10.09.2009.

22 Martin Nettesheim: Entmündigung der Politik, in: Frankfurter Allgemeine Zeitung, 27.08.2009; dagegen Hillgruber: Die besseren Europäer, 2009.

23 Lenz: Ausbrechender Rechtsakt, 2009.

24 Christian Tomuschat: The Ruling of the German Constitutional Court on the Treaty of Lisbon, in: German Law Journal 8/2009, S. 1260-1261.

25 Lenz: Ausbrechender Rechtsakt, 2009. 
grationsbremsen“26 und eine Schwächung der europäischen Rechtsordnung. ${ }^{27}$ Und aus Frankreich äußert Alfred Grosser sogar, das Urteil werfe Fragen zur Ernsthaftigkeit von Deutschlands europäischem „Commitment“ auf, und sieht den 30. Juni 2009 als einen schwarzen Tag in Europas Geschichte. ${ }^{28}$

Nicht nachzugehen ist nachfolgend dem nicht spekulationsfreien Fragenkreis, ob die verbal und inhaltlich stärkere Hervorhebung von verfassungsrechtlichen Integrationsvoraussetzungen und -grenzen durch das Lissabon-Urteil im Vergleich zum Maastricht-Urteil mit pauschalen Gesichtspunkten in der öffentlichen Diskussion seit 1993 in Verbindung zu bringen ist wie etwa mit einer als zu weitgehend, auch kulturell identitätsbedrohend und sich in unkontrolliertem Selbstlauf vollziehend gefühlten Vertiefung der Europäischen Union, ${ }^{29}$ einer als unübersichtlich und entgrenzend empfundenen Erweiterung der Europäischen Union $^{30}$ oder einer als sich wandelnd erscheinenden Rolle Deutschlands. ${ }^{31}$ Vielmehr ist das Urteil in seinen rechtlichen Weichenstellungen und Folgerungen für die Integrationspolitik und sind sodann deren Optionen in Augenschein zu nehmen. Erläuternd ist vorwegzuschicken: Ein Gerichtsurteil, will es überzeugen, muss sowohl den gesetzlichen Textgrundlagen und der Systemrationalität des für die Entscheidung maßgeblichen Rechtsbereichs genügen (hier des GG) als auch den zu bewertenden Sachverhalt zutreffend aufnehmen (hier die drei deutschen Gesetze in Verbindung mit dem VvL) und überdies (auch als Urteil eines Verfassungsorgans, das dessen ungeachtet ein Gericht ist ${ }^{32}$ ) judikativ vorgehen. Für das BVG war deshalb das Zustimmungsgesetz und dadurch inzident der VvL lediglich der Sachverhalt, auf den es die in seiner Auslegungszuständigkeit stehenden einschlägigen Vorschriften des GG anzuwenden hatte. Umgekehrt kann nationales Recht einschließlich des Verfassungsrechts für den Europäischen Gerichtshof (EuGH), namentlich in einem Vertragsverletzungsverfahren, ${ }^{33}$ zum Sachverhalt werden, auf den es die in seiner Auslegungszuständigkeit ste-

26 So namentlich die SWP-Analyse von Peter Becker/Andreas Maurer: Deutsche Integrationsbremsen. Folgen und Gefahren des Karlsruher Urteils für Deutschland und die EU, Stiftung Wissenschaft und Politik: SWP-Aktuell 41, Juli 2009.

27 Ebenda. Zu dieser Gefahr auch Peter-Christian Müller-Graff: L'arrêt de Karlsruhe sur le traité de Lisbonne, in: Regards sur l'économie allemande 92/2009, S. 5-12, hier 10ff.

28 Alfred Grosser: The Federal Constitutional Court's Lisbon Case: Germany's "Sonderweg" - An Outsider's Perspective, in: German Law Journal 8/2009, S. 1263-1266, hier S. 1263.

29 Vgl. z.B. Rupert Scholz: Einführung, in: Rupert Scholz (Hrsg.): Deutschland auf dem Weg in die Europäische Union: Wieviel Eurozentralismus - wieviel Subsidiarität?, Köln 1994, S. 29-34, 31ff.; Joachim Starbatty: Die Administration: Was und wie bestimmt „Brüssel“ tatsächlich? Wege gegen bürokratische Ausuferung, in: Rupert Scholz (Hrsg.): Deutschland auf dem Weg in die Europäische Union: Wieviel Eurozentralismus - wieviel Subsidiarität?, Köln 1994, S. 44-55, hier S. 50ff.; dazu und zu den zur Unitarisierung gegenläufigen Faktoren Peter-Christian Müller-Graff: Unitarisierung in der Europäischen Union?, in: Klaus Grupp/Ulrich Hufeld (Hrsg.): Recht - Kultur - Finanzen. Festschrift für Reinhard Mußgnug zum 70. Geburtstag am 26. Oktober 2005, Heidelberg 2005, S. 311-328, hier S. 317ff., 325ff.

30 Zu dieser Frage z.B. Karel De Gucht: Enlargement: Past and Future, in: Astrid Epiney/Marcel Haag/Andreas Heinemann (Hrsg.): Die Herausforderung von Grenzen. Festschrift für Roland Bieber, Baden-Baden 2007, S. 485-492.

31 So eine Andeutung von Lenz: Ausbrechender Rechtsakt, 2009; so die Frage von Grosser: The Federal Constitutional Court's Lisbon Case, 2009; vgl. als Bestandsaufnahme und zu Perspektiven der Verantwortung Deutschlands in Europa Peter-Christian Müller-Graff (Hrsg.): Deutschlands Rolle in der Europäischen Union, 2. Aufl., Baden-Baden 2009.

32 „Deutlicher als den Status des Verfassungsorgans spricht das GG das BVerfG als Gericht an“ liest man in der Kommentierung zum Bundesverfassungsgericht in Michael Sachs (Hrsg.): Grundgesetz. Kommentar, 3. Aufl., München 2003, Art. 93 Rdz. 9.

33 Derzeit Art. 226 des Vertrags zur Gründung der Europäischen Gemeinschaft in der konsolidierten Fassung des Vertrages von Nizza (EGV), in: Amtsblatt der EU, Nr. C 321 E vom 29. Dezember 2006, S. 37-186; projektiert: Art. 258 des Vertrags über die Arbeitsweise der Europäischen Union in der konsolidierten Fassung des Vertrags von Lissabon (AEUV), in: Amtsblatt der EU, Nr. C 115 vom 9. Mai 2008, S. 47-199. 
henden Normen des Europarechts anzuwenden hat. Das Terrain und die Zuständigkeit des BVG zu letztverbindlicher Entscheidung liegen daher nicht im Europarecht, sondern im deutschen Verfassungsrecht. Die nachfolgende Erörterung und Beurteilung der Grundlinien des Urteils erfolgt im Einzelnen aus fünf Fragewinkeln: seiner Lokation im Koordinatensystem der ausdrücklichen integrationsrechtlichen Aussagen des GG, seiner verfassungsrechtlichen Weichenstellungen, seiner Folgerungen für die verfassungsrechtliche Beurteilung des VvL, seiner Szenarienbildung hypothetischer Konfliktsituationen zwischen Integrationsentwicklung und deutschem Verfassungsrecht mit der (Selbst-)Definition der Rolle des BVG sowie schließlich seiner integrationspolitischen Konsequenzen. Hierbei ist insbesondere nach den Optionen europäischer Integrationspolitik im Blick auf die vom BVG hervorgehobenen verfassungsrechtlichen Aussagen zu fragen.

\section{Die Lokation des Lissabon-Urteils im Koordinatensystem der ausdrücklichen integra- tionsrechtlichen Aussagen des Grundgesetzes: der konditionierte Integrationsauftrag}

Ausgangspunkt einer Bewertung der integrationsrelevanten verfassungsrechtlichen Weichenstellungen und Folgerungen des Lissabon-Urteils sind die Textaussagen des GG zum Verhältnis von Verfassung und europäischer Integration.

\section{Die europäische Integration als ein Staatsziel der deutschen Verfassung}

Die textlich ausdrückliche Öffnung des GG zur europäischen Integration seit seiner Urfassung des Jahres 1949 ist bekannt. Die Präambel bekundet, dass das deutsche Volk von dem Willen beseelt ist, als gleichberechtigtes Mitglied in einem vereinten Europa dem Frieden der Welt zu dienen. Gerade auch dazu ermächtigte von Anfang an der allgemeine Integrationsartikel (Art. 24 GG) ${ }^{34}$ den Bund, ,durch Gesetz Hoheitsrechte auf zwischenstaatliche Einrichtungen [zu] übertragen“. Der nach der Wiedervereinigung eingefügte EUspezielle Integrationsartikel (Art. 23 GG) konkretisiert diese Ermächtigung als einen Staatszielauftrag ${ }^{35}$ mit den Worten, dass die Bundesrepublik Deutschland zur Verwirklichung eines vereinten Europas bei der Entwicklung der Europäischen Union mitwirkt, die demokratischen, rechtsstaatlichen, sozialen und föderativen Grundsätzen und dem Grundsatz der Subsidiarität verpflichtet ist und einen dem GG im Wesentlichen vergleichbaren Grundrechtsschutz gewährt ${ }^{36}$ (sogenannte Strukturanforderungen an die Europäische Union). Das Lissabon-Urteil bestätigt ausdrücklich diese Öffnung der staatlichen Herrschaftsordnung für das friedliche Zusammenwirken der Nationen und die europäische Integration. ${ }^{37}$ Es erkennt klar und geschichtsbewusst, dass das GG sich ,,von einer selbstgenügsamen und selbstherrlichen Vorstellung souveräner Staatlichkeit“ löst und Souveränität als völkerrechtlich gebundene und geordnete Freiheit auffasst, ${ }^{38}$ wobei es souveräne Staatlichkeit als einen befriedeten Raum und die darin gewährleistete Ordnung auf der Grundlage individueller Freiheit und kollektiver Selbstbestimmung definiert. ${ }^{39}$ Das Urteil zieht aus der Öffnung der staatlichen Hoheitsordnung des GG zutreffend die seit Langem erkannte und unausweichliche Konsequenz, dass die ,dadurch ermöglichte neue Gestalt politischer Herrschaft [...] nicht schematisch den innerstaatlich geltenden verfassungsstaatlichen Anforderungen [unterliegt]

34 Vgl. dazu im Einzelnen z.B. die Kommentierung von Rudolf Streinz, in: Michael Sachs (Hrsg.): Grundgesetz. Kommentar, 3. Aufl., München 2003, Art. 24 Rdz. 1ff.

35 Rudolf Streinz, in: Michael Sachs (Hrsg.): Grundgesetz. Kommentar, 3. Aufl., München 2003, Art. 23 Rdz. 10.

36 Art. 23 Abs. 1 S. 1 GG.

37 Vgl. Rdz. 219 bis 225, 229, 230 des Urteils.

38 Vgl. Rdz. 223 des Urteils.

39 Vgl. Rdz. 224 des Urteils. 
und [...] deshalb nicht umstandslos an den konkreten Ausprägungen des Demokratieprinzips in einem Vertrags- oder Mitgliedstaat gemessen werden [darf] “. ${ }^{40}$ Es erkennt gleichermaßen zutreffend die erst jüngst wieder von Werner Link hervorgehobene integrationspolitische Paradoxie, ${ }^{41}$ dass nur der das erforderliche Maß an Gestaltungsmöglichkeiten gewinnt, der sich ,aus Einsicht in die Notwendigkeit friedlichen Interessenausgleichs und in die Möglichkeiten gemeinsamer Gestaltung" freiwillig und gleichberechtigt mit Staaten desselben Wertefundaments bindet. ${ }^{42}$ Hierbei versteht es wie schon das Maastricht-Urteil die Europäische Union in eigener (nicht dem GG entstammender) Begrifflichkeit als „Staatenverbund“ konzipiert. $^{43}$

\section{Voraussetzungen integrationsgerichteter Änderungen der Hoheitsordnung im Text des Grundgesetzes}

Zum Verhältnis zwischen dem Mitwirkungsauftrag an der europäischen Integration und der staatlichen Hoheitsordnung nimmt das GG in seinem Text bekanntlich neben der Verankerung der inhaltlichen Strukturanforderungen an die Europäische Union eine Festlegung zu den legitimatorischen Voraussetzungen der Übertragung von Hoheitsrechten auf die Europäische Union vor. ${ }^{44}$ Beinhaltet diese, wie regelmäßig, eine inhaltliche Änderung oder Ergänzung des Grundgesetzes oder eine Ermöglichung solcher Änderungen oder Ergänzungen, so sind die formellen und materiellen Bedingungen einer Verfassungsänderung zu erfüllen, also insbesondere das Erfordernis einer Zweidrittelmehrheit in Bundestag und Bundesrat, ${ }^{45}$ sowie das absolute Änderungsverbot (sogenannte Ewigkeitsgarantie) bestimmter Wesenszüge des GG einzuhalten. ${ }^{46} \mathrm{Zu}$ letzteren zählen die Bundesstaatlichkeit, die grundsätzliche Mitwirkung der Länder bei der Gesetzgebung, die Achtung der Menschenwürde und der dazu ,unentbehrlichen Substanz elementarer Grundrechte ${ }^{\text {47 }}$ sowie die Staatsstrukturprinzipien des Art. 20 GG (Demokratie, Rechts- und Sozialstaatlichkeit, Republik, Bundesstaat). Zu ihnen fügt das Lissabon-Urteil mittels des Demokratieprinzips ausdrücklich die „,souveräne Staatlichkeit“ Deutschlands hinzu ${ }^{48}$ (dazu unten). Die von der Ewigkeitsgarantie erfassten Wesenszüge bezeichnet das BVG als „Verfassungsidentität“. ${ }^{49}$ Der Schwerpunkt der Begründung des Lissabon-Urteils ist einerseits der Bestimmung der daraus ${ }^{50}$ und aus den Strukturanforderungen an die Europäische Union abzuleitenden Vorgaben und Grenzen der Integration ${ }^{51}$ und andererseits der Überprüfung des Zustimmungsgesetzes ${ }^{52}$ und der Begleitgesetzgebung zum $\mathrm{VvL}^{53}$ anhand dieser Kriterien gewidmet.

40 Vgl. Rdz. 219 des Urteils.

41 Werner Link: Auf dem Weg zu einem neuen Europa. Herausforderungen und Antworten, Baden-Baden 2006, S. $21 \mathrm{ff}$.

42 Vgl. Rdz. 220 bis 222, 247 des Urteils.

43 Vgl. Rdz. 229 des Urteils; zur terminologischen Problematik dieses vom Maastricht-Urteil kreierten Begriffs Meinhard Hilf: Die Europäische Union und die Eigenstaatlichkeit ihrer Mitgliedstaaten, in: Peter Hommelhoff/ Paul Kirchhof (Hrsg.): Der Staatenverbund der Europäischen Union, Heidelberg 1994, S. 75-86, hier S. 75-76.

44 Ermächtigung in Art. 23 Abs. 1 S. 2 GG.

45 Art. 23 Abs. 1 S. 3 GG i.V.m. Art. 79 Abs. 2 GG.

46 Art. 23 Abs. 1 S. 3 GG i.V.m. Art. 79 Abs. 3 GG.

47 Vgl. Rdz. 217 des Urteils.

48 Vgl. Rdz. 216, 228 des Urteils.

49 Vgl. Rdz. 218 des Urteils.

50 Vgl. Rdz. 217 des Urteils.

51 Vgl. Rdz. 226 bis 272 des Urteils.

52 Vgl. Rdz. 273 bis 399 des Urteils.

53 Vgl. Rdz. 400 bis 420 des Urteils. 


\section{Die verfassungsrechtlichen Weichenstellungen des Lissabon-Urteils: der zugrunde- gelegte Prüfungsmaßstab}

Das BVG baut seine Beurteilung der drei ihm vorgelegten Gesetze im Wesentlichen ${ }^{54}$ jedoch nicht auf der Mitwirkungsklausel des Art. 23 GG, sondern auf dem verfassungsrechtlichen Prüfungsmaßstab des Wahlrechts zum Bundestag (Art. 38 GG) und damit auf dem Demokratieprinzip auf, ${ }^{55}$ verbindet dieses mit weiteren verfassungsrechtlichen Grundsätzen und verknüpft diese wiederum mit verfassungsrechtlichen Bedingungen und Grenzen der Integration. Es widmet der Erarbeitung seines Prüfungsmaßstabs auffälligerweise rund 40 Prozent seines Wertungsteils. ${ }^{56}$

Das individuelle Wahlrecht zum Bundestag als Ausdruck des Demokratieprinzips und des Prinzips personaler Freiheit als Prüfungsmaßstab

Der Prüfungsansatz an dem subjektiven Recht, an der Wahl der Abgeordneten des Deutschen Bundestages teilzunehmen, ${ }^{57}$ mag angesichts der Existenz der im Jahre 1992 neu geschaffenen ausdrücklichen objektiven Maßstabsnorm des Art. 23 Abs. 1 GG und dessen Prüfungsprogramm für die Mitwirkung der Bundesrepublik bei der Entwicklung der Europäischen Union überraschen. Dies erklärt sich jedoch zunächst aus dem zugrunde liegenden Verfahren. In ihm waren unter anderem ${ }^{58}$ Verfassungsbeschwerden eingelegt worden. Dieser Weg steht einem Beschwerdeführer aber bekanntlich nur mit der Behauptung offen, durch die öffentliche Gewalt (einschließlich der Legislative) in einem seiner Grundrechte oder einem grundrechtsgleichen Recht verletzt worden zu sein..$^{59}$ Das Urteil nimmt dies zum Anlass, entscheidende verfassungsrechtliche Weichenstellungen bereits in der Frage der Zulässigkeit der Individualbeschwerde anzudeuten oder vorzunehmen ${ }^{60}$ und damit individualbezogen zuzuschneiden, und baut diese dann in der Prüfung deren Begründetheit weitausgreifend aus. ${ }^{61}$

Der Ansatz beim Wahlrecht zum Bundestag erfolgt in Nachfolge zum Maastricht-Urteil. ${ }^{62}$ In ihm hatte das BVG bekanntlich erstmals die Zulässigkeit der Individualbeschwerde gegen ein Zustimmungsgesetz zu einem europäischen Integrationsvertrag eröffnet, die auf die Verletzung des Wahlrechts gestützt war, ${ }^{63}$ und damit keineswegs nur Zustimmung gefunden. ${ }^{64}$ Zugrunde liegen zwei Überlegungen: erstens, dass das Wahlrecht als elementarer Bestandteil des Demokratieprinzips nicht nur den förmlichen Wahlakt umfasst, sondern als Anspruch auf die für die Staatsordnung des GG konstitutive ${ }^{65}$ demokratische Selbstbestimmung nach dem Mehrheitsprinzip und auf freie und gleiche Teilhabe an der öffentlichen Ge-

54 Ein weiterer, aber vergleichsweise eingegrenzter Ansatz liegt in dem verbundenen Organstreitverfahren in dem Sonderbereich des wehrverfassungsrechtlichen Parlamentsvorbehalts; vgl. Rdz. 204-205 des Urteils.

55 Vgl. Rdz. 208 des Urteils.

56 Vgl. Rdz. 172 bis 271 (von Rdz. 172 bis 420) des Urteils.

57 Vgl. Rdz. 174, 208 des Urteils.

58 Verbunden war ein Organstreitverfahren.

59 Art. 93 Abs. 1 Nr. 4a GG.

60 Vgl. Rdz. 172ff. des Urteils.

61 Vgl. Rdz. 208-272 des Urteils.

62 BVerfGE 89, 155, 171f.

63 Ebenda.

64 Vgl. statt vieler z.B. die seinerzeitigen Kritiken des ehemaligen BVG-Richters Helmut Steinberger: Anmerkungen zum Maastricht-Urteil des Bundesverfassungsgerichts, in: Peter Hommelhoff/Paul Kirchhof (Hrsg.): Der Staatenverbund der Europäischen Union, Heidelberg 1994, S. 25-38, hier S. 25-27; und des heutigen BVG-Richters (Erster Senat) Brun-Otto Bryde: Das Maastricht-Urteil des Bundesverfassungsgerichts. Konsequenzen für die weitere Entwicklung der europäischen Integration, Tübingen 1993, S. 4ff. 
walt ${ }^{66}$ (die das BVG jetzt sogar in der unveränderbaren Garantie der Menschenwürde verankert $^{67}$ ) auch das Recht zum Einfluss darauf, wie die Staatsgewalt auf Bundesebene ausgeübt wird; 68 und zweitens, dass das Wahlrecht durch Veränderung der Organisation der Staatsgewalt im Falle eines ,wesentlich[en] ",Substanzverlust[s] demokratischer Gestaltungsmacht" des Bundestages verletzt sein könne, ${ }^{69}$ da der Wahlakt seinen Sinn verlöre, ,wenn das gewählte Staatsorgan nicht über ein hinreichendes Maß an Aufgaben und Befugnissen verfügte“ (,konkrete Verantwortung für das Handeln des Staatsverbandes“), weil „,die durch die Wahl bewirkte Legitimation von Staatsgewalt und Einflussnahme auf deren Ausübung“ durch Kompetenzverlagerung auf die europäische Ebene so entleert wäre, dass das Demokratieprinzip verletzt würde. ${ }^{70}$ Diese sehr weitgehende Aufladung des Wahlrechts und die mit dem Kriterium des „hinreichende[n] Maß[es]“ oder ,,ausreichenden Raums zur politischen Gestaltung "71 unbestimmte Aussage wird vom Lissabon-Urteil bestätigt. ${ }^{72}$ Sie enthält zwei Prämissen: erstens, dass mit der Wahl zum Bundestag neben der Legitimation der Staatsgewalt ,auch dirigierender Einfluss genommen [wird], wie diese ausgeübt wird“; 73 zweitens eine tendenziell engführende Verknüpfung zwischen Demokratieprinzip und staatlichen Sachkompetenzen (,,konkrete Verantwortung“ im Unterschied zu Wahl- und Prozeduralkompetenzen im Sinne einer ,, abstrakten , Gewährleistungsverantwortung ' für das hoheitliche Handeln anderer Herrschaftsverbände“;74 gemeint: Europäische Union). Hier stellen sich Fragen. Inwieweit lässt sich ein signifikant höheres Maß von „dirigierende[m]“ Einfluss des Einzelnen über den Wahlakt auf die „konkrete Verantwortung“ gegenüber dem Einfluss auf die „Gewährleistungsverantwortung“ substanziieren, insbesondere, wenn man vergleichend - bei der „konkreten Verantwortung“ an Entscheidungen denkt, die im Bundesstaat vom Bundestag, der sich aus Wahlkreisvertretern und Listenvertretern zusammensetzt, gemeinsam mit dem Bundesrat, der sich aus Regierungsvertretern der Länder zusammensetzt, getroffen werden? Und inwieweit lässt sich substantiieren, dass und ab welcher Schwelle das Wahlrecht als individuelles Selbstbestimmungs- und Machtrecht entleert ist, wenn das direkt gewählte Organ nicht selbst ein ,hinreichendes“ Maß an Sachfragen entscheidet, sondern die über die gewählte Parlamentsmehrheit bestimmte Regierung über diese Sachfragen im Rat der Europäischen Gemeinschaft/Europäischen Union mitentscheidet? Die staatliche Mitwirkung an Maßnahmen der europäischen Ebene kommt bei diesem Ansatz wertungsmäßig nur abgestuft in den Blick. Man kann zweifeln, dass eine derartige Abstufung dem Integrationsauftrag des GG mit seinem erweiterten Horizont der Hoheitsausübung gerecht wird.

Allerdings wird diese Weitung vom Urteil dann aufgenommen, indem es die auf das Wahlrecht gestützte Beschwerdebefugnis auch auf die Behauptung von Defiziten der demokratischen Legitimation der Europäischen Union ausweite ${ }^{75}$ und damit einer konzeptionell starren Engführung der Legitimations- und Einflusskraft der Wahl auf die Ausübung nationaler sachgebietlicher Verbandskompetenzen vorbeugt. Stimmig dazu kommt in der Tiefe der Urteilsbegründung auch das Erfordernis der „,maßgeblichen“ Einflussnahme der Bun-

66 Vgl. Rdz. 208, 210, 211 des Urteils.

67 Vgl. Rdz. 211 des Urteils.

68 Vgl. Rdz. 208 des Urteils.

69 Vgl. Rdz. 210 des Urteils.

70 Vgl. Rdz. 175, 208, 210, 246 des Urteils in Nachfolge zu BVerfGE 89, 155, 172.

71 Vgl. Rdz. 249 des Urteils.

72 Vgl. Rdz. 175 des Urteils.

73 Vgl. Rdz. 174 des Urteils.

74 Vgl. Rdz. 175 des Urteils.

75 Vgl. Rdz. 177, 261 des Urteils. 
desregierung auf europäische Entscheidungsverfahren ins Spiel ${ }^{76}$ und blitzt der Gedanke der Erweiterung des verfassungsrechtlichen „Föderalmodells um eine überstaatlich kooperative Dimension“ auf. ${ }^{77}$ Allerdings löst dies noch nicht die Frage nach Ableitung und Inhalt des abstrakten Kriteriums eines verbleibenden ,hinreichende[n] Maß[es]“ der ,konkrete[n] Verantwortung “ im Sinne nationaler Verbandskompetenzen innerhalb des Integrationsauftrages des GG noch die Frage nach der Legitimation des BVG zu der darin potenziell liegenden Bevormundung offener demokratischer Willensbildungsprozesse in Bundestag, Bundesrat und Bundesregierung.

Die Verbindung des individuellen Wahlrechts zu weiteren Grundsätzen (Souveränität, Verfassungsidentität und Sozialstaatlichkeit)

Darüber hinaus bejaht das BVG in einer weiteren Weichenstellung die auf das Wahlrecht gestützte Beschwerdebefugnis nunmehr auch bei Behauptung des Verlusts der ,souveränen Staatlichkeit“ der Bundesrepublik ${ }^{78}$ und der „Verfassungsidentität““ ${ }^{79}$ Denn es bewertet eine Umbildung der Bundesrepublik zu einem Gliedstaat eines europäischen Bundesstaates als „Identitätswechsel“ der Bundesrepublik und ihrer Verfassungsordnung, mit der eine Ablösung des GG einhergehe. Zu dieser sei aber nicht die durch das GG verfasste Gewalt befugt, sondern allein die zu achtende verfassungsgebende Gewalt des Volkes, ${ }^{80}$ an der wiederum (wegen Art. 146 GG) ein „Teilhaberecht des wahlberechtigten Bürgers“ bestehe. ${ }^{81}$ Dies ist ein kühner Bogenschlag zur Verfassungsbeschwerde. Inhaltlich hat diese Folgerung zur Voraussetzung, dass das GG eine ,souveräne Staatlichkeit Deutschlands“ als unveränderbare „Verfassungsidentität“ garantiert. ${ }^{82}$ Beides sind allerdings keine Eigenbegriffe des GG in der Ewigkeitsgarantie (Art. 79 Abs. 3 GG). Tatsächlich ist hier auch ein Dissens zu denjenigen zu registrieren, die, wie Carl Otto Lenz aus dem optionsoffenen Integrationsauftrag des GG erklären, dass das politische Ziel eines europäischen Bundesstaats, wie es etwa von Konrad Adenauer bis Helmut Kohl vertreten wurde (und wie es strukturell wohl kaum identisch zu einem nationalen Bundesstaatsmodell mit starker Zentralgewalt und vergleichsweise schwächeren Gliedstaaten gedacht $w^{8}{ }^{83}$ ), nicht als grundgesetzwidrige Forderung verstanden wurde. ${ }^{84}$ Auch das Maastricht-Urteil war in kluger Zurückhaltung nicht so weit gegangen. ${ }^{85}$ Dass das Urteil zu einem derart grundsätzlichen, aber unaktuellen und eher ältlich wirkenden ${ }^{86}$ Thema apodiktisch mit grober Bundesstaatsbegrifflichkeit, die ohnehin nicht zur Entwicklung des innovativen Modells der Vergemeinschaftung passt, in einem Verfahren Stellung nimmt, in dem es darauf nicht ernstlich ankommt, erstaunt. Dies weicht

76 Vgl. Rdz. 246 des Urteils.

77 Vgl. Rdz. 277 des Urteils.

78 Vgl. Rdz. 178-180, 208, 216-218 des Urteils.

79 Rdz. 218 des Urteils.

80 Diesen „Appell an die [...] Revolution“ hält Nettesheim: Entmündigung der Politik, 2009, für ,,verfassungsstaatlich unzulässig", doch bleibt die Begründung dieser Aussage unklar.

81 Vgl. Rdz. 179, 218, 228 des Urteils.

82 Ausdrücklich Rdz. 216 des Urteils: „Mit der sogenannten Ewigkeitsgarantie wird die Verfügung über die Identität der freiheitlichen Verfassungsordnung selbst dem verfassungsändernden Gesetzgeber aus der Hand genommen. Das Grundgesetz setzt damit die souveräne Staatlichkeit Deutschlands nicht nur voraus, sondern garantiert sie auch."

83 Auf diesen Aspekt wies zu Recht schon Bryde: Das Maastricht-Urteil, 1993, S. 15, nach dem Maastricht-Urteil hin.

84 Vgl. Lenz: Ausbrechender Rechtsakt, 2009, der sich hierzu vor allem auf den Parlamentarischen Rat bezieht.

85 Vgl. Bryde: Das Maastricht-Urteil, 1993, S. 12-13.

86 Bildhaft sieht Schönberger: Lisbon in Karlsruhe, 2009, S. 1217 eine Reanimation des Geistes einer Vision zum Zweck seines Ausschlusses aus dem GG. 
von der richterlichen Primäraufgabe ab, nur diejenigen Fragen zu entscheiden, um die es im konkreten Fall geht. ${ }^{87}$ Andererseits werden dadurch zwar politische Argumentationsoptionen erschwert (etwa die vor einigen Jahren von Karl Lamers vorgeschlagene deutsch-französische Föderation), nicht aber wird das Entdeckungsverfahren der Integration beendet. In den Händen des französischen Conseil constitutionnel hat das Prinzip der nationalen Souveränität, soweit ersichtlich, bis heute keinen Integrationsschritt blockiert. ${ }^{88}$

Schließlich erfolgt eine weitere Weichenstellung darin, dass die auf das Wahlrecht gestützte Beschwerdebefugnis auch bejaht wird, wenn eine Verletzung des Sozialstaatsprinzips auf die Behauptung gestützt wird, die demokratischen sozialpolitischen Gestaltungsmöglichkeiten des Bundestages würden durch den Zuständigkeitenausbau der Europäischen Union derart beschränkt, dass er die sich aus dem GG ergebenden „Mindestanforderungen des Sozialstaatsprinzips nicht mehr erfüllen könne“. ${ }^{89}$ Dies ist erneut eine Weitung, die aber im Grunde nur eine besonders hervorgehobene einzelne sachpolitische Konkretisierung des Ansatzes des BVG beim Erfordernis ,hinreichender“ nationaler Verbandskompetenzen beinhaltet.

\section{Abstrakte verfassungsrechtliche Bedingungen und Grenzen der Integration}

Da in einer Verfassungsordnung viele ihrer abstrakten Einzelelemente über deren jeweilige Teilaspekte miteinander in Verbindung gebracht werden können, gelangt das BVG über den Einstieg beim individuellen Wahlrecht über die Prinzipien der Demokratie, der ,,souveränen Staatlichkeit“ und der „Verfassungsidentität“ schließlich zu ihrerseits abstrakten und wertungsoffenen Bedingungen und Grenzen der europäischen Integration ${ }^{90}$ und bereitet mit seiner hierzu entwickelten Begrifflichkeit seinem eigenen Aufgaben- und Arbeitsumfang und seiner Selbstherausforderung potenziell fruchtbaren Boden. Denn es gebraucht in der grundlegenden Weichenstellung der verfassungsrechtlichen Ermächtigungsbedingungen zur Integration ${ }^{91}$ ausnahmslos Termini, die nicht dem Text des GG entstammen, sondern Eigenschöpfungen oder übernommene Kreationen sind: Wahrung der ,souveränen Staatlichkeit“ auf der Grundlage eines ,Integrationsprogramms“ nach dem Prinzip der ,,begrenzten Einzelermächtigung“92 (begrifflicher Ursprung im Europarecht) und der Achtung der ,verfassungsrechtlichen Identität als Mitgliedstaaten“ (Ähnlichkeit zu Art. 6 Abs. 3 EUV ${ }^{93}$ ) sowie Erhalt der Fähigkeit der Mitgliedstaaten ,zu selbstverantwortlicher politischer und sozialer Gestaltung der Lebensverhältnisse“. Es schafft sich dadurch argumentative Freiräume.

87 Nicht alles, was Parteien in einem Verfahren vortragen, ist für eine Sachentscheidung bedeutsam. Überdies wird gerade für die Verfassungsgerichtsbarkeit ,eine der wenigen und gerade darum besonders fundamentalen Grenzen“ darin gesehen, dass es ,nur reaktiv auf Fragen antworten darf, die ihm zulässigerweise vorgelegt worden sind“; so Bryde: Das Maastricht-Urteil, 1993, S. 4-5.

88 Vgl. zur Rechtsprechung des Conseil constitutionnel bis 1998 Peter-Christian Müller-Graff/Anja Reichel: Die europäische Integration aus der Sicht der Rechtsprechung nationaler Verfassungsgerichte, in: Mathias Jopp/ Andreas Maurer/Heinrich Schneider (Hrsg.): Europapolitische Grundverständnisse im Wandel. Analysen und Konsequenzen für die politische Bildung, Bonn 1998, S. 365-418, hier S. 378ff., 384ff., $397 f$.

89 Vgl. Rdz. 181 bis 183 des Urteils.

90 Vgl. Rdz. 226 bis 272 des Urteils.

91 Vgl. Rdz. 226 des Urteils.

92 Der Begriff entstammt zwar dem Europarecht (vgl. jetzt auch projektierter Art. 5 Abs. 1 und 2 des Vertrags über die Europäische Union in der konsolidierten Fassung des Vertrags von Lissabon (EUV-Liss.), in: Amtsblatt der EU, Nr. C 115 vom 9. Mai 2008, S. 13-45), doch ist nicht auszuschließen, dass er als Verfassungsbegriff und damit spezifischer normativer Zweckbegriff einen spezifischen Eigeninhalt gewinnen kann.

93 Vertrag über die Europäische Union in der konsolidierten Fassung des Vertrages von Nizza (EUV), in: Amtsblatt der EU, Nr. C 321 E vom 29. Dezember 2006, S. 5-36. Dort allerdings ,,nationale Identität ihrer Mitgliedstaaten". 
Übersichtlicher und naheliegender wäre ein judikativer Ansatz beim Wortlaut und Prüfungsprogramm des EU-Integrationsauftrags (Art. 23 Abs. 1 GG) gewesen.

Im Einzelnen entfaltet das Urteil eine lange Reihe von (überwiegend grenzziehenden) Ableitungen: dass das GG nicht zur Ausgestaltung der demokratischen Selbstbestimmung auf europäischer Ebene nach dem deutschen Bundesstaatsmodell verpflichte, sondern Abweichungen von den Organisationsprinzipien innerstaatlicher Demokratie zulässig seien ${ }^{94}$ (das ist konsequent zu Art. 23 GG); dass es bei der Integration in eine freiheitliche Gemeinschaft keine ,der verfassungsrechtlichen Begrenzung und Kontrolle entzogene Unterwerfung“ (das Wort assoziiert einen seltsamen Opferblick) noch den Verzicht auf die ,eigene Identität" verlange; ${ }^{95}$ dass es nicht dazu ermächtige, ,,durch einen Eintritt in einen Bundesstaat das Selbstbestimmungsrecht des Deutschen Volkes in Gestalt der völkerrechtlichen Souveränität Deutschlands aufzugeben“; 96 dass es die Mitwirkung an einem ,Staatenverbund“ zulasse, den es nunmehr definiert als ,eine enge, auf Dauer angelegte Verbindung souverän bleibender Staaten, die auf vertraglicher Grundlage öffentliche Gewalt ausübt, deren Grundordnung jedoch allein der Verfügung der Mitgliedstaaten unterliegt und in der die Völker - das heißt die staatsangehörigen Bürger - der Mitgliedstaaten die Subjekte demokratischer Legitimation bleiben“;97 dass es keine Unterschreitung des durch die Ewigkeitsgarantie geschützten „Mindeststandard[s]“ zulasse; ${ }^{98}$ dass es die überstaatliche Gemeinschaftsgewalt auf eine von den Staaten abgeleitete Autonomie begrenze; ${ }^{9}$ dass es die Übertragung der Kompetenz-Kompetenz auf die Europäische Union untersage; ${ }^{100}$ dass es die sachliche Begrenzung und prinzipielle Widerruflichkeit der Einräumung vermehrter Zuständigkeiten an die Europäische Union fordere und ein Prinzip der umkehrbaren Selbstbindung gelte ${ }^{101}$ (letzteres, wenn verstanden als einseitiges Kündigungsrecht ohne weitere Voraussetzungen, steht im Widerspruch zum derzeitigen Gemeinschafts-/Unionsrecht ${ }^{102}$ ); dass es das Prinzip der begrenzten Einzelermächtigung als Ausdruck der staatsverfassungsrechtlichen Grundlegung der Unionsgewalt fordere; 103 dass es die „Verfassungsidentität“ „unübertragbar und insoweit integrationsfest“ stelle; ${ }^{104}$ dass es eine ,hinreichend[e]“ Bestimmtheit des „Integrationsprogramm[s]“ fordere;105 dass es eine effektive Sicherung der Integrationsverantwortung der gesetzgebenden Körperschaften in Deutschland mittels Zustimmungsgesetzgebung bei ratifikationslosen Änderungen des Primärrechts durch die Unionsorgane fordere; ${ }^{106}$ dass es kein unbegrenztes Vertrauen in die konstruktive Kraft des Integrationsmechanismus habe und daher eine „exzeptionell[e]“ ,äußere“ Kontrolle der Unionsorgane in Fragen der Auslegung des Vertragsrechts fordere ${ }^{107}$ (aus welcher Norm des

94 Vgl. Rdz. 227 des Urteils.

95 Vgl. Rdz. 228 des Urteils.

96 Vgl. ebenda.

97 Vgl. Rdz. 229 des Urteils.

98 Vgl. Rdz. 230 des Urteils.

99 Vgl. Rdz. 231, 232 des Urteils.

100 Vgl. Rdz. 233 des Urteils.

101 Vgl. ebenda.

102 Vgl. Art. 51 EUV und Art. 312 EGV; statt vieler Matthias Pechstein, in: Rudolf Streinz (Hrsg.): EUV/EGV. Kommentar, München 2003, Art. 49 EUV Rdz. 17, Art. 51 EUV Rdz. 2; Juliane Kokott, in: Rudolf Streinz (Hrsg.): EUV/EGV. Kommentar, München 2003, Art. 312 EGV Rdz. 4ff.; Albrecht Weber, in: Hans von der Groeben/Jürgen Schwarze (Hrsg.): EUV/EGV. Kommentar, 6. Aufl., München 2003, Art. 312 EGV Rdz. 7ff., 9 und Art. 51 EUV Rdz. 1.

103 Vgl. Rdz. 234 des Urteils.

104 Vgl. Rdz. 235 des Urteils.

105 Vgl. Rdz. 236 des Urteils.

106 Vgl. Rdz. 236, 239, 242 des Urteils (mit schwammigen Formulierungen).

107 Vgl. Rdz. 238 des Urteils. 
GG ergibt sich das?); dass es innerhalb der deutschen Jurisdiktion ermögliche, die Integrationsverantwortung im Fall „ersichtliche[r] Grenzüberschreitungen“ durch die Europäische Union und zur Wahrung des ,unantastbaren Kerngehalts der Verfassungsidentität des Grundgesetzes" einzufordern; ${ }^{108}$ dass es im Gefolge einer derartigen ,Ultra-vires '-Kontrolle und „Identitätskontrolle“ zulasse, dass „Gemeinschafts- oder künftig Unionsrecht in Deutschland für unanwendbar erklärt" werde. ${ }^{109}$ Erstaunlich an dieser letztgenannten Konsequenz ist schon die Beanspruchung einer derartigen Rechtsfolgenmacht ohne ausdrückliche gesetzliche Grundlage. ${ }^{110}$ Denn es geht um die im Gewaltenteilungssystem (auch im internationalen Vergleich) keineswegs selbstverständliche Möglichkeit, den Entscheidungen demokratisch legitimierter politischer Organe gerichtlich ihre Wirkung zu nehmen anstatt die politischen Organe auf die Inkompatibilität eines Aktes mit der Verfassung hinzuweisen und diesen die Remedur zu überlassen. Auf die Problematik dieses Anspruchs im Licht des verfassungsrechtlichen Integrationsauftrags und des Europarechts ist weiter unten einzugehen.

Schließlich konkretisiert das BVG in Konsequenz seines Ansatzes die ,demokratischen Grundsätze“, denen die Ausgestaltung der Europäischen Union sowohl in Art und Umfang der Übertragung von Hoheitsrechten als auch in organisatorischer und verfahrensrechtlicher Ausgestaltung entsprechen müsse. Dies erfolgt in zwei Richtungen, ${ }^{111}$ deren erste zu den Verbandskompetenzen der Bundesrepublik zurückführt.

Denn erstens dürfe die Integration nicht zu einer Aushöhlung des demokratischen Systems in Deutschland führen. ${ }^{112}$ Deshalb treffe die deutschen Verfassungsorgane eine dauerhafte Integrationsverantwortung, ${ }^{113}$ die im Interesse des Erhalts eines ,gestaltenden Einfluss[es] auf die politische Entwicklung in Deutschland“ voraussetze, dass der „Bundestag eigene Aufgaben und Befugnisse von substantiellem politischem Gewicht behält oder die ihm politisch verantwortliche Bundesregierung maßgeblichen Einfluss auf europäische Entscheidungsverfahren auszuüben vermag“. ${ }^{114}$ Hier stellt das BVG den zusätzlichen Gedanken ein, dass die Supranationalisierung die Bildung eines unmittelbar auf das Volk zurückgehenden Mehrheitswillens erschwere, und folgert daraus, dass das Demokratieprinzip der Hoheitsrechtsübertragung inhaltliche Grenzen setze, die nicht bereits aus der Unverfügbarkeit der staatlichen Souveränität folgten. ${ }^{115}$ Es stellt dazu fest, dass das GG zwar nicht den Verbleib einer von vornherein bestimmbaren Summe von Hoheitsrechten oder bestimmter Arten von Hoheitsrechten fordere, ${ }^{116}$ unterstreicht jedoch als cantus firmus erneut, dass das europäische Zusammenwirken nicht derart verwirklicht werden dürfe, ,,dass in den Mitgliedstaaten kein ausreichender Raum zur politischen Gestaltung der wirtschaftlichen, kulturellen, sozialen Lebensverhältnisse mehr bleibt“.117 Die davon erfassten wesentlichen Bereiche substanziiert das BVG beispielhaft aufzählend als ,unter anderem die Staatsbürgerschaft, das zivile und militärische Gewaltmonopol, Einnahmen und Ausgaben einschließ-

108 Vgl. Rdz. 240 des Urteils.

109 Vgl. Rdz. 240-241 des Urteils.

110 Der Wortlaut des $\S 78$ des Bundesverfassungsgerichtsgesetzes (Nichtigerklärung von Gesetzen; dazu Malte Graßhof, in: Dieter Umbach/Thomas Clemens/Franz-Wilhelm Dollinger (Hrsg.): Bundesverfassungsgerichtsgesetz. Mitarbeiterkommentar und Handbuch, Tübingen 2005, § 78; Lechner/Rüdiger: Bundesverfassungsgerichtsgesetz, 2006, § 78) deckt diese Rechtsfolge nicht.

111 Vgl. Rdz. 244 bis 272 des Urteils.

112 Vgl. Rdz. 244 des Urteils.

113 Vgl. Rdz. 245 des Urteils.

114 Vgl. Rdz. 246 des Urteils.

115 Vgl. Rdz. 247 des Urteils.

116 Vgl. Rdz. 248 des Urteils.

117 Vgl. Rdz. 249 des Urteils. 
lich der Kreditaufnahme sowie die für die Grundrechtsverwirklichung maßgeblichen Eingriffstatbestände [..., kulturelle Fragen wie die Verfügung über die Sprache, die Gestaltung der Familien- und Bildungsverhältnisse, die Ordnung der Meinungs-, Presse- und Versammlungsfreiheit oder der Umgang mit dem religiösen oder weltanschaulichen Bekenntnis“..118 Eine konzeptionelle Rückführung dieser Bereiche auf die klassischen Staatsmerkmale erfolgt nicht, scheint aber durch. Unbegründet bleibt, warum sich das BVG derart exponiert, aber letztlich allgemein, in Fragen der Integrationspolitik begibt, die zuallererst von den demokratisch legitimierten politischen Organen zu behandeln sind. ${ }^{119}$ Beiläufig gerät damit das währungsunionale Konvergenzkriterium der jährlichen Nettoneuverschuldung in ein schräges Licht. ${ }^{120}$ Das Urteil vertieft in diesem Teil der Kriterienentwicklung zunächst ohne ausdrückliche Bezugnahme auf den VvL, aber erkennbar von dessen Neuerungen inspiriert, abstrakt (und mit in sich durchaus tragfähigen Begründungen) fünf sensible Bereiche staatlicher Selbstgestaltungsfähigkeit: ${ }^{121}$ die Strafrechtspflege, den Einsatz der Bundeswehr, das Budgetrecht, das Sozialstaatsprinzip und die Möglichkeit zur Verwirklichung im eigenen Kulturraum, wobei hier verdienstvollerweise die Sprache hervorgehoben wird.

Als zweite Dimension der demokratischen Anforderungen legt das Urteil sodann als Grundlinie fest, dass die supranationale öffentliche Gewalt „für sich genommen“ grundlegende demokratische Anforderungen nicht verfehlen dürfe und diese Anforderungen wiederum vom Umfang der übertragenen Hoheitsrechte und vom Verselbständigungsgrad europäischer Entscheidungsverfahren abhingen. ${ }^{122}$ Diese flexible Verknüpfung ist einleuchtend. Das Urteil findet hierbei unter teilweisem Rückgriff auf das Maastricht-Urteil zu einer zweigeteilten kompetenzabhängigen ,Solange'-Formel zur Legitimation des Handelns der Europäischen Union: ${ }^{123}$ zum einen zur Abgrenzung vom Maßstab staatlicher Legitimation (,Solange die europäische Zuständigkeitsordnung nach dem Prinzip der begrenzten Einzelermächtigung in kooperativ ausgestalteten Entscheidungsverfahren unter Wahrung der staatlichen Integrationsverantwortung besteht und solange eine ausgewogene Balance der Unionszuständigkeiten und der staatlichen Zuständigkeiten erhalten bleibt, kann und muss die Demokratie der Europäischen Union nicht staatsanalog ausgestaltet sein.“), zum anderen zu den positiven Voraussetzungen (,,Solange und soweit das Prinzip der begrenzten Einzelermächtigung in einem Verbund souveräner Staaten mit ausgeprägten Zügen exekutiver und gouvernementaler Zusammenarbeit gewahrt bleibt, reicht grundsätzlich die über nationale Parlamente und Regierungen vermittelte Legitimation der Mitgliedstaaten aus, die ergänzt und abgestützt wird durch das unmittelbar gewählte Europäische Parlament.“).

\section{Die Folgerungen des Lissabon-Urteils für die verfassungsrechtliche Beurteilung des durch den VvL neugestalteten Primärrechts der Europäischen Union}

In Anwendung seiner Kriterien kommt das BVG in ausführlicher Begründung mit vielen Widerhaken zu dem Ergebnis, dass das Zustimmungsgesetz zum VvL ,nach Maßgabe der Gründe" mit den Anforderungen des Grundgesetzes, insbesondere mit dem Demokratieprinzip, vereinbar ist. ${ }^{124}$ Diese Bewertung gründet auf der Gesamteinschätzung, dass das Wahl-

$118 \mathrm{Vgl}$. ebenda.

119 So auch Nettesheim: Entmündigung der Politik, 2009.

120 Vgl. Becker/Maurer: Deutsche Integrationsbremsen, 2009, S. 4.

$121 \mathrm{Vgl}$. Rdz. 252 bis 261 des Urteils.

122 Vgl. Rdz. 261 bis 272 des Urteils.

123 Vgl. Rdz. 262, 272 des Urteils.

124 Vgl. Rdz. 273 des Urteils. 
recht zum Bundestag nicht verletzt ist, weil auch nach Inkrafttreten des VvL ,das Deutsche Volk in Bund und Ländern nach wie vor über wesentliche politische Sachverhalte " bestimmt und mit ,der Wahl des deutschen Kontingents von Abgeordneten des Europäischen Parlaments [...] für das Wahlrecht der Bundesbürger eine ergänzende Mitwirkungsmöglichkeit im europäischen Organsystem eröffnet“" ist. ${ }^{125}$ Darin spiegelt sich die anfängliche Verknüpfung der Wahl zum Bundestag und dessen Sachkompetenzen (,konkrete Verantwortung“) unter Abstufung des Einflusses auf die Europäische Union über die vom Bundestag eingesetzte Bundesregierung wider. Außerdem beinhaltet die Formulierung an dieser Stelle eine (wohl redaktionell unbemerkte) Engführung der demokratischen Legitimationsvermittlung für die europäische Ebene auf das Europäische Parlament unter Ausklammerung des Rates, der aber später richtigerweise einbezogen wird. Überdies erklärt das BVG mit der Formulierung „,nach Maßgabe der Gründe“ einen warnenden, aber eben gerade wegen der Bezugnahme auf die Gründe des Urteils alles andere als klaren Vorbehalt. Das BVG bewertet das Legitimationsniveau der Europäischen Union nach dem VvL im Hinblick auf deren Zuständigkeitsumfang und politische Verselbstständigung als ,,noch“126 bedingt grundgesetzkonform. Die (orakelartige) Bedingung liegt darin, dass das Prinzip der begrenzten Einzelermächtigung ,über das in den Verträgen vorgesehene Maß hinaus“ zu sichern ist. Es stützt seine Bewertung auf drei tiefschürfende, teils sehr verschlungene Gedankenketten, die die Neuerungen des $\mathrm{VvL}$ im Wesentlichen verständig rezipieren.

BVG: Das nicht staatsanaloge, aber auch nicht staatsanalog erforderliche, gleichwohl in sich defizitäre Legitimationsniveau der Europäischen Union im $V v L$

Erstens wertet das BVG das Handeln der Europäischen Union unter dem Gesichtspunkt seiner Legitimation derart, dass die Europäische Union auch durch den VvL zwar noch keine Ausgestaltung erreiche, die dem Legitimationsniveau einer staatlich verfassten Demokratie entspreche, ${ }^{127}$ ein solches aber mangels staatsanalogen Aufbaus von Aufgaben und Herrschaftssystem der Europäischen Union auch nicht erforderlich, ${ }^{128}$ gleichwohl aber in sich defizitär sei. ${ }^{129}$ Damit bewältigt das BVG zunächst das von ihm zutreffend analysierte Problem, dass die vom VvL fortgeführte Zusammensetzung des Europäischen Parlaments nach degressiv proportionalen Länderkontingenten ${ }^{130}$ nicht der demokratischen Grundregel der wahlrechtlichen Erfolgschancengleichheit folgt. ${ }^{131}$ Denn diese Regel verbindet es nur mit einem Volk, ${ }^{132}$ nicht aber mit einem supranationalen Vertretungsorgan der Völker, ${ }^{133}$ in dem ,politische Machtlagen der Mitgliedstaaten“ ähnlich wie im Rat im Sinne des Gedankens repräsentativer Demokratie ${ }^{134}$ „nur vermittelt“ (trotz Direktwahl?) repräsentiert werden. ${ }^{135}$ Aus dem Gedanken der Staatenunion (also der vertraglichen Einigung über die Machtverteilung) erklärt das BVG auch den in der Tat vorhandenen Wertungswiderspruch

125 Vgl. Rdz. 274 des Urteils.

126 Vgl. Rdz. 275 des Urteils.

127 Vgl. Rdz. 276, 289ff., 295 des Urteils.

128 Vgl. Rdz. 278 des Urteils.

129 Vgl. Rdz. 289 des Urteils.

130 Projektierter Art. 14 Abs. 2 S. 3 EUV-Liss.; derzeit Art. 190 Abs. 2 EGV.

131 Vgl. Rdz. 279 des Urteils; zu weiteren Schwächen des Europäischen Parlaments vgl. Peter M. Huber: Wer das Sagen hat, in: Frankfurter Allgemeine Zeitung, 10.09.2009.

132 Vgl. Rdz. 279, 282 des Urteils.

133 Vgl. Rdz. 279, 284 des Urteils.

134 Projektierter Art. 10 Abs. 1 EUV-Liss.

135 Vgl. Rdz. 286 des Urteils. 
zwischen dem Postulat einer Bürgerunion und der Unterscheidung des Repräsentationsgewichts der Unionsbürger nach Staatsangehörigkeiten ${ }^{136}$ im Europäischen Parlament. ${ }^{137}$

Obwohl das BVG sich damit vom Maßstab staatlicher Demokratieanforderungen gelöst zu haben scheint, prüft es selbstwidersprüchlich dann doch, ob die von ihm durchaus als legitimationssteigernd anerkannten ${ }^{138}$ Neuerungen des VvL (ergänzende partizipative, assoziative und direkte Demokratiegehalte, doppelt-qualifizierte Mehrheit im Rat, Einbeziehung nationaler Parlamente) ,das - gemessen an staatlichen Demokratieanforderungen - bestehende Defizit der europäischen Hoheitsgewalt“ aufwiegen, und verneint dies. ${ }^{139}$ In dieser Passage mit durchaus bedenkenswerten Relativierungen des Demokratiegehalts der partizipativen und assoziativen Elemente ${ }^{140}$ führt die strenge Orientierung am Maßstab des - gerade nicht als anwendbar erklärten - staatlichen Herrschaftsmodells indes zu einer gedanklichen und argumentativ zirkulären Einengung. Der Einfluss der Wähler auf die Zusammensetzung des Europäischen Parlaments, des Rates und des Europäischen Rates in ihrem komplexen Zusammenspiel wird nicht vertieft analysiert. ${ }^{141}$ Die Qualifizierung der Kommission mit der „Funktion einer [...] europäischen Regierung“ (bereits nach geltendem Recht!) und die daraus unklar angedeuteten Folgerungen für künftige Entwicklungen ${ }^{142}$ sind mangels offengelegter Kriterien des Verständnisses von ,Regierung' nicht nachvollziehbar beziehungsweise unverständlich. Vor allem aber wird das zu einer supranationalen Organisation passfähige neue Legitimationsmodell der doppelten Mehrheit im Rat nicht hinreichend gewürdigt. ${ }^{143}$ Denn wenn in einer „Staatenunion“ mit einem starken Organ der Vertretung der Regierungen und einem strukturell als Völkervertretung nach degressiver Proportionalität konzipierten Europäischen Parlament das demokratische Wahlgleichheitsgebot mangels eines Unionsvolkes nicht erfüllbar ist, jedoch auf eine überzeugende demokratische Legitimation auch auf Unionsebene nicht verzichtet werden kann, dann bedarf es speziell für die unionalen Entscheidungsverfahren eines anderen Modells als des staatlich konzipierten „Wahlakts des Individuums“, ${ }^{144}$ um demokratische Legitimation zu vermitteln. Daher ist die Neuerung der doppelten Mehrheit ein gerade demokratisch höchst achtbares, die ,Summe [der] Betroffenen“ staatlich gegliedert einbeziehendes und der Supranationalität des „Staatenverbundes“ angemessenes innovatives Legitimationsmodell. Es tritt ergänzend zu der ohnehin vorhandenen einzelstaatlichen demokratischen Legitimation der Regierungsvertreter hinzu. Hier wird die Einschätzung des BVG dem Erfordernis der angemessenen Anpassung des Demokratieprinzips an Entscheidungsabläufe in einer supra-

136 Diese Unterscheidung trifft jedenfalls im Grundsatz zu, ist aber über das Wahlrecht von Unionsbürgern anderer Mitgliedstaaten im Wohnsitzstaat (projektiert Art. 22 Abs. 2 EUV-Liss.; derzeit Art. 19 Abs. 2 EUV) um die Wohnsitzkomponente modifiziert.

137 Vgl. Rdz. 287 des Urteils.

138 Vgl. Rdz. 294 des Urteils.

139 Vgl. Rdz. 289ff., 295 des Urteils.

140 Projektierte Art. 11 Abs. 1 bis 3 EUV-Liss.: öffentlicher Ansichtenaustausch, Dialog ,,mit den repräsentativen Verbänden und der Zivilgesellschaft", Anhörungen der Betroffenen.

141 Lenz: Ausbrechender Rechtsakt, 2009.

142 Vgl. Rdz. 297 des Urteils.

143 Kurze Passage in Rdz. 292 des Urteils zum „Korrektiv der Bevölkerungsmehrheit“ gegenüber dem Grundsatz der Staatengleichheit als eines ,,weiteren Zurechnungssubjekts“, ,,das aus den Völkern der Mitgliedstaaten der Union besteht, wobei nicht auf die Unionsbürger als Subjekte politischer Herrschaft, sondern auf die Einwohner der Mitgliedstaaten als Ausdruck der Vertretungsmächtigkeit des Ratsvertreters des betreffenden Mitgliedstaats Bezug genommen wird." Das BVG sieht dadurch das demokratische Wahlgleichheitsgebot nicht als erfüllt an, denn die ,demokratische Legitimation politischer Herrschaft wird bei der Wahlgleichheit und dem unmittelbaren parlamentarischen Repräsentationsmechanismus auch in Parteiendemokratien in der Kategorie des Wahlakts des Individuums gedacht und nicht am Maßstab der Summe Betroffener beurteilt."

144 Vgl. Rdz. 292 des Urteils. 
nationalen Organisation als zwar abgeleiteter, aber eben mit supranationaler Eigenausprägung konzipierter Herrschaftsform nicht gerecht. Sie ist sogar kontraproduktiv zu der legitimatorisch wichtigen Errungenschaft der doppelten Mehrheit. ${ }^{145}$

BVG: Das Prinzip der begrenzten und „kontrolliert ausgeübten“ Einzelermächtigung der Europäischen Union und der Erhalt der Staatlichkeit der Mitglieder der Europäischen Union im $V v L$

Aus der Fortgeltung des „Verbundprinzips“ im VvL, der in seiner Grundkonzeption nicht vom VVE abweicht (wie eine missverständliche Formulierung des BVG insinuieren könnte ${ }^{146}$ ), folgert das Gericht in sich konsequent (weil erneut das Demokratieprinzip mit dem Umfang der Sachkompetenzen des Bundestages verkoppelnd), dass es für die Vereinbarkeit des VvL mit dem GG darauf ankomme, dass Kompetenzausstattung und Kompetenzausübung der Europäischen Union dem - nunmehr vom BVG aus dem Europarecht in das Verfassungsrecht rezipierten - Prinzip der „,begrenzten und kontrolliert ausgeübten Einzelermächtigung “147 - mithin dem Erfordernis der „,hinreichenden Bestimmtheit des Integrationsprogramms“148 - genügen: und damit der Wahrung der ,verfassungsrechtliche[n] und politische[n] Identität der volldemokratisch organisierten Mitgliedstaaten“ und ihrer „Verantwortung für die grundlegende Richtung und Ausgestaltung der Unionspolitik“ und dem Erhalt der „,staatlichen Souveränität“. ${ }^{149}$ Das BVG sieht diese Voraussetzungen als vom VvL erfüllt, weil sich dessen Bestimmungen aus seiner Sicht in dieser Weise auslegen lassen. ${ }^{150}$ Die Begründung vollzieht sich in länglicher Auseinandersetzung mit der (für die Gestalter des VvL oder etwa dem französischen oder britischen Verständnis des VvL doch etwas erstaunlichen) grundlegenden Frage, ob die Bundesrepublik (und damit implizit auch etwa Frankreich oder Großbritannien) durch den VvL die souveräne völkerrechtssubjektive Staatlichkeit verliere. Folgerichtig werden die drei klassischen Kriterien der Staatlichkeit von Georg Jellinek (Staatsgewalt, Staatsgebiet, Staatsvolk) durchgeprüft, wobei es im Wesentlichen um die Staatsgewalt geht. ${ }^{151}$ Angewandt auf die derzeitige Europäische Gemeinschaft/Europäische Union belegen diese Maßstäbe bekanntlich ihre Nicht-Staatlichkeit; ${ }^{152}$ der VvL ändert daran nichts. Angewandt auf die Bundesrepublik, erkennt das BVG zu Recht und wenig überraschend, dass es bei ihrer Staatlichkeit bleibt. Es folgert dies aus vier ausführlichen Überlegungen, deren letzte freilich erheblichen Sprengstoff bergen: (1.) Die Neuerungen der Zuständigkeitsordnung (Verteilung und Abgrenzung ${ }^{153}$ ) bleiben auf der Spur des Prinzips der begrenzten Einzelermächtigung ${ }^{154}$ und europarechtlicher Schutzmechanismen ${ }^{155}$ (zutreffend); (2.) die neuen Vertragsänderungsregeln (insbesondere vereinfachtes

145 So auch Becker/Maurer: Deutsche Integrationsbremsen, 2009, S. 7.

146 In Rdz. 298 des Urteils: „Gerade nach dem Scheitern des europäischen Verfassungsprojekts ist mit dem Vertrag von Lissabon hinreichend deutlich geworden, dass dieses Verbundprinzip weiter gilt." Das war auch beim VVE klar. Die meisten seiner projektierten Neuerungen prägen im Übrigen den VvL.

147 Vgl. Rdz. 234, 298 des Urteils.

148 Vgl. Rdz. 236 des Urteils.

149 Vgl. Rdz. 298 des Urteils.

$150 \mathrm{Vgl}$. ebenda.

151 Vgl. Rdz. 299 bis 343 des Urteils.

152 Vgl. zur Anwendung dieser Kriterien auf die Europäische Gemeinschaft/Europäische Union Peter-Christian Müller-Graff: Verfassungsziele der EG/EU, in: Manfred Dauses (Hrsg.): Handbuch des EU-Wirtschaftsrechts, München 2000, A I Rdz. 60ff.

153 Projektierte Artt. 2 bis 6 EUV-Liss.

154 Vgl. Rdz. 301 bis 303 des Urteils.

155 Vgl. Rdz. 304 bis 305 des Urteils. 
Änderungsverfahren, ${ }^{156}$ Brückenklauseln, ${ }^{157}$ Reform der Flexibilitätsklausel ${ }^{158}$ ) stehen dem nicht entgegen ${ }^{159}$ (zutreffend; zu deren innenpolitischen Ausfüllungsvoraussetzungen unten); (3.) das neue Austrittsrecht „,macht erstmals das bestehende Recht jedes Mitgliedstaats zum Austritt aus der Europäischen Union [...] sichtbar"160 (die Annahme, es gebe derzeit ein jederzeitiges Austrittsrecht, widerspräche geltendem Unionsrecht, ${ }^{161}$ doch erledigt sich diese Kontroverse mit dem Inkrafttreten des $\mathrm{VvL}^{162}$ ); und (4.) der Fortbestand souveräner Staatsgewalt ,wird durch das dem Bundesverfassungsgericht zustehende Letztentscheidungsrecht [...] geschützt". 163

Letzteres ist ein wuchtiger Klimmzug des BVG in die Arena der Europapolitik, dessen europarechtliche Kompatibilität unten zu erörtern ist. Der Anspruch auf ein Letztentscheidungsrecht (im Verhältnis zur Europäischen Union) kann bei Fragen der Auslegung von Gemeinschaftsrecht oder der Anwendbarkeit von Sekundärrecht im Extremfall eine Kooperations- und Loyalitätsverweigerung 164 beinhalten, konkreter: eine Verweigerung des Vorlageverfahrens an den EuGH oder der Befolgung einer Entscheidung des EuGH. Man kann in diesem Postulat den selbstständig festgelegten Preis des BVG für die verfassungsrechtliche Freigabe des VvL erkennen. Ihn vermag wegen der Begründung des BVG aus dem GG der Gesetzgeber letztlich wohl nur mit verfassungsändernder Mehrheit zu überwinden beziehungsweise zu senken. ${ }^{165}$ Es liegt aber in der Hand des EuGH, diesen Anspruch durch kluge Rechtsprechung sowohl in Einzelfällen als auch prinzipienevolutiv mittels einer ,Cassis '-analogen Rechtsprechung zu den Immanenzgrenzen des Europarechts (im ,Cassis ‘Bereich zu zwingenden mitgliedstaatlichen Allgemeininteressen als Grenzen der Marktgrundfreiheiten ${ }^{166}$ ) für den Schutz der „Verfassungsidentität“ gemeinschafts-/unionsrechtlich zu entschärfen.

\section{BVG: Der Erhalt eigener Aufgaben und Zuständigkeiten von hinreichendem Gewicht für den Deutschen Bundestag}

Schließlich bejaht das BVG, dass dem Bundestag nach dem VvL „eigene Aufgaben und Zuständigkeiten von hinreichendem Gewicht“ in Umfang und Substanz als ,,rechtlich[e] und praktisch[e] Voraussetzung für eine lebendige Demokratie“ verbleiben. Es begründet diese Schlussfolgerung damit, dass die neu projektierten Zuständigkeiten der Europäischen Union ,jedenfalls bei der gebotenen Auslegung“ keine „staatsbegründenden Elemente“ seien, die

156 Projektierter Art. 48 Abs. 6 EUV-Liss.; ferner projektierte Art. 42 Abs. 2 UAbs. 1 EUV-Liss.; Artt. 25 Abs. 2 , 218 Abs. 8 UAbs. 2 S. 2, 223 Abs. 1 UAbs. 2, 262 und 311 Abs. 3 AEUV.

157 Projektierte Art. 48 Abs. 7 EUV-Liss., Art. 81 Abs. 3 UAbs. 3 AEUV; ferner projektierte Art. 31 Abs. 3 EUVLiss., Artt. 153 Abs. 2 UAbs. 4, 192 Abs. 2 UAbs. 2, 312 Abs. 2 UAbs. 2, 333 Abs. 1 und 2 AEUV.

158 Projektierter Art. 352 EUV-Liss. (derzeit Art. 308 EGV).

159 Vgl. Rdz. 306 bis 328 des Urteils.

160 Vgl. Rdz. 329 bis 330 des Urteils.

161 Vgl. Art. 51 EUV und Art. 312 EGV; Matthias Pechstein, in: Rudolf Streinz (Hrsg.): EUV/EGV. Kommentar, München 2003, Art. 49 EUV Rdz. 17, Art. 51 EUV Rdz. 2; Juliane Kokott, in: Rudolf Streinz (Hrsg.): EUV/EGV. Kommentar, München 2003, Art. 312 EGV Rdz. 4ff.; Albrecht Weber, in: Hans von der Groeben/Jürgen Schwarze (Hrsg.): EUV/EGV. Kommentar, 6. Aufl., München 2003, Art. 312 EGV Rdz. 7ff., 9 und Art. 51 EUV Rdz. 1.

162 Projektierter Art. 50 EUV-Liss.

163 Vgl. Rdz. 299, 331 bis 343 des Urteils.

$164 \mathrm{Zu}$ der vom Masstricht-Urteil abweichenden Nichterwähnung des Kooperationsverhältnisses Calliess: Unter Karlsruher Totalaufsicht, 2009.

165 Allerdings bestehen hier Fragen zu den Rechtswirkungen für den Gesetzgeber im Einzelnen; vgl. dazu allgemein Lechner/Zuck: Bundesverfassungsgerichtsgesetz, 2006, § 31; Michael Sachs: Verfassungsprozessrecht, Frankfurt am Main 2004, S. 168ff.

166 Grundlegend EuGH Rs. 120/78 (Rewe/Bundesmonopolverwaltung für Branntwein), Urteil vom 20.02.1979, Slg. 1979, 649. 
auch in einer verfassungsrechtlichen Gesamtschau die souveräne Staatlichkeit der Bundesrepublik nicht verletzten. ${ }^{167}$ Das BVG kommt zu diesem Ergebnis mittels Entfaltung seines teils detaillierten Auslegungsverständnisses der neuen Zuständigkeiten der Union in den Bereichen der justiziellen Zusammenarbeit in Strafsachen ${ }^{168}$ und Zivilsachen, ${ }^{169}$ der Außenwirtschaftsbeziehungen ${ }^{170}$ und der gemeinsamen Verteidigung ${ }^{171}$ und einer abrundenden Betrachtung der sozialpolitischen Dimension, ${ }^{172}$ ohne auf diese hier im Einzelnen eingehen zu können. Damit deutet das BVG überdeutlich die Möglichkeit seiner künftigen Nachkontrolle der Kompetenzausübung an. Es artikuliert dazu vorsorglich sein eigenes Verständnis der ,gebotenen Auslegung" im Sinne einer engen Auslegung der einschlägigen neuen Vertragsbestimmungen, ${ }^{173}$ das in der Sache ein breitflächiges Monitum an die Politik und Vertragsauslegung der Bundesorgane sowie der europäischen Organe einschließlich des EuGH beinhaltet. Dieser Aspekt ist bei den integrationspolitischen Konsequenzen näher zu behandeln.

\section{Die Szenarienbildung des Lissabon-Urteils: hypothetische Konflikte zwischen Inte- grationsentwicklung und deutschem Verfassungsrecht und die Rolle des Bundesver- fassungsgerichts}

Ungewöhnlich zur judikativen Kernaufgabe des Entscheidens aktueller Streitfragen enthält die Begründung des Lissabon-Urteils neben den Ausführungen zum Prüfungsmaßstab des GG und seiner Anwendung auf den VvL als forciert wirkenden roten Faden immer wieder die Erörterung hypothetischer künftiger Konflikte zwischen Unionsentwicklung und Grundgesetz und der Rolle des BVG in diesen. Das BVG sieht eine ,verfassungsrechtlich bedeutsame Spannungslage“ zum Prinzip der begrenzten Einzelermächtigung und zur parlamentarischen Integrationsverantwortung, wenn das Primärrecht durch „Organe“ der Europäischen Union ,,verändert oder erweiternd ausgelegt wird“, ${ }^{174}$ folgert daraus das Erfordernis einer ,sei es auch nur sehr zurückgenommene[n] und sich als exzeptionell verstehende[n] äußere[n] Kontrolle“ darüber, wie das Vertragsrecht ausgelegt wird, ${ }^{175}$ und bringt sich damit schließlich selbst als Kontrolleur ${ }^{176}$ im Sinne einer „Reservekompetenz “177 ins Spiel. In dieser (jedenfalls normativ-verbalen, wiewohl nicht notwendig faktischen) Selbststärkung des Gerichts im Gang der europäischen Integration liegt ein Hauptcharakteristikum und zugleich Hauptproblem der gesamten umfänglichen Begründung. Sie und ihre Folgen geben Anlass zu mehreren Fragen. Das Gericht erweitert im Vergleich zum Maastricht-Urteil zunächst das Problemfeld hypothetischer Konflikttypen und wendet sich dann seiner eigenen Rolle zu.

\section{Erweiterung des Kreises hypothetischer Konfliktsituationen durch das Lissabon-Urteil}

$\mathrm{Zu}$ den Reibungsflächen des Gemeinschaftshandelns mit dem grundgesetzlichen Grundrechtsschutz (BVG-Urteile: ,Solange I‘;178 entspannt durch ,Solange II ${ }^{` 179}$ ) und dem Prinzip

167 Vgl. Rdz. 351 des Urteils.

168 Projektierte Artt. 82ff. AEUV.

169 Projektierter Art. 81 AEUV.

170 Projektierte Artt. 206ff. AEUV.

171 Projektierte Artt. 42ff. EUV-Liss.

172 Vgl. Rdz. 392 bis 400 des Urteils.

173 Vgl. Rdz. 351ff. des Urteils.

174 Vgl. Rdz. 238 des Urteils.

175 Vgl. Rdz. 238, 334 des Urteils.

176 Vgl. Rdz. 240 des Urteils.

177 Vgl. Rdz. 341 des Urteils.

178 BVerfGE 37, 271ff.

179 BVerfGE 73, 339ff. 
der begrenzten Einzelermächtigung (,ultra vires ‘; Maastricht-Urteil ${ }^{180}$ ) wird nunmehr, wie gezeigt, auch eine Konfliktmöglichkeit mit der „Verfassungsidentität“ und verfassungsgebenden Gewalt der Mitgliedstaaten als Herren der Verträge prononciert. ${ }^{181}$ Die Voraussetzungen, aufgrund deren europäisches Sekundärrecht in Deutschland unanwendbar sein kann, ${ }^{182}$ bleiben allerdings wenig bestimmt und werden vermehrt. Zum ,ausbrechenden Rechtsakt"183 im Sinne eines ,Ultra-vires'-Handelns ${ }^{184}$ kommen beispielsweise terminologisch hinzu: ${ }^{185}$ „ersichtliche Grenzüberschreitung“, ,unantastbarer Kerngehalt der Verfassungsidentität“, ,nicht erlangbarer Rechtsschutz auf Unionsebene“ (im Ergebnis?) und anderes mehr. ${ }^{186}$ Diese hochabstrakten und wertungsoffenen Begriffe beinhalten für die Gestaltung einer einzelnen konkreten sekundärrechtlichen Einzelbestimmung mehr orakelartige Unklarheit als dirigierende Orientierung.

\section{Die Selbstpositionierung des Bundesverfassungsgerichts}

Zur Bewältigung derartiger hypothetischer Konfliktsituationen stellt das BVG darauf ab, dass die Auslegung des Unionsrechts ,äußerer“ Kontrolle bedarf ${ }^{187}$ und entwickelt zusätzlich die Auffassung, dass die Verfassungsidentität der Bundesrepublik bei fortschreitender Integration anders als durch eine „Identitätskontrolle“ seitens des BVG nicht gewahrt werden kann. ${ }^{188}$ Diese generelle Aussage ist aus verschiedenen Gründen diskussionsbedürftig.

Erstens unter dem Gesichtspunkt der ,Kontrolle‘. Denn der Rechtsschutz gegen Kompetenzüberschreitungen der politischen Gemeinschaftsorgane ist primärrechtlich ausdrücklich der Gemeinschaftsgerichtsbarkeit zugewiesen (prozedural namentlich: Nichtigkeitsklage, ${ }^{189}$ Vorabentscheidungsverfahren, ${ }^{190}$ Inzidentkontrolle ${ }^{191}$ ). Dadurch kann es auch zum Schutz der „nationalen Identität“ der Mitgliedstaaten im Sinne des EU-Vertrages ${ }^{192}$ kommen: derzeit implizit ${ }^{193}$ und nach dem VvL direkt. ${ }^{194}$ Die Notwendigkeit von Rechtsschutz gegen Kompetenzüberschreitungen steht außer Zweifel. Dies zeigte sich namentlich aus Anlass des fadenscheinigen Versuchs von Europäischem Parlament und Rat, bei der allgemeinen Tabakwerberichtlinie Gesundheitspolitik als Marktintegrationspolitik zu maskieren, um eine Kompetenz für Rechtsangleichung zu usurpieren. Er wurde aufgrund einer Nichtigkeitsklage der Bundesrepublik vom EuGH durchkreuzt. ${ }^{195}$ Auffälligerweise wird die Gemeinschaftsgerichtsbarkeit aber im Passus des Lissabon-Urteils zum Rechtsschutz nur ohne Nennung und Würdigung ihrer Leistungen gestreift (,Wenn Rechtsschutz auf Unionsebene nicht

180 BVerfGE $89,155 \mathrm{ff}$

181 Vgl. Rdz. 234f. des Urteils.

182 Vgl. Rdz. 241, 339 des Urteils.

183 BVerfGE 89, 155.

184 Vgl. Rdz. 240, 338 des Urteils: „Grenzen der eingeräumten Hoheitsrechte“.

185 Vgl. Rdz. 240 des Urteils.

186 Des Weiteren z.B. „Integrationsverantwortung“ (Rdz. 236); ,,vertragsausdehnende Auslegung [...] durch die Gemeinschaftsgerichtsbarkeit, die einer unzulässigen autonomen Vertragsänderung gleichkomm[t]“ (Rdz. 338); ,ersichtlich[er]“ Mangel des ,konstitutiven Rechtsanwendungsbefehl[s]“ (Rdz. 339).

187 Vgl. Rdz. 238 des Urteils.

188 Vgl. Rdz. 240 des Urteils.

189 Derzeit Art. 230 EGV und Art. 35 Abs. 6 EUV; projektiert Art. 263 AEUV.

190 Derzeit Art. 234 EGV und Art. 35 Abs. 3 EUV; projektiert Art. 267 AEUV.

191 Derzeit Art. 241 EGV; projektiert Art. 277 AEUV.

192 Derzeit Art. 6 Abs. 3 EUV; projektiert Art. 4 Abs. 2 EUV-Liss.

193 In der Bestimmung der Zuständigkeit des EuGH führt der derzeitig maßgebliche Art. 46 EUV nicht Art. 6 Abs. 3 EUV auf.

194 Projektierter Art. 4 Abs. 2 EUV-Liss.

195 EuGH Rs. C-376/98 (Deutschland/Parlament und Rat), Urteil vom 05.10.2000, Slg. 2000, I-8419. 
zu erlangen ist [...]“196) und in ihrer Aufgabe ${ }^{197}$ vor allem derart einbezogen, dass dadurch den Mitgliedstaaten ,das Recht zur Prüfung der Einhaltung des Integrationsprogramms nicht entzogen sein kann“. 198 Das Urteil spricht in der Frage ,dynamischer Integrationsentwicklung“ sogar pauschal von den „Organen“ der Europäischen Union, die ,äußerer“ Kontrolle bedürfen, ${ }^{199}$ ohne hierbei den EuGH auszunehmen. Eine derartige Herabstufung von Funktion und Leistung des EuGH bei der Rechtswahrung erstaunt. Überdies bleibt völlig offen, wieso , anders als“ gerade durch das BVG die „Strukturen souveräner Mitgliedstaaten“ (Plural!) nicht gewahrt werden können. Ist dies nicht ein primäres Thema demokratischer Politik?

Zweitens vermittelt die Akzentsetzung auf eine ,äußere“ Kontrolle den seltsamen Eindruck, dass das BVG die Europäische Union als ein Gebilde betrachtet, mit dem die Bundesrepublik nicht verzahnt zu sein scheint. Terminologisch wirkt dies als Distanzierung zum europäischen Gemeinschaftsgedanken und zum Integrationsauftrag des GG.

Drittens ist der Begriff der „Verfassungsidentität“ ein abstrakter Sammelbegriff, den das GG in seinem Text nicht ausdrücklich führt. Während sich die genetische Kodierung eines Individuums ähnlich wie ein Fingerabdruck als unverwechselbar identitätsstabil präzise bestimmen lässt und dies in Grenzen auch für dessen soziale Kodierung möglich sein mag, kann die Identitätsbestimmung der Verfassungsordnung im Rahmen der Ewigkeitsgarantie des GG nur mittels ihrer sehr abstrakten, überwiegend wertungsoffenen und dadurch potenziell beweglichen Elemente gelingen, wodurch auch die Schutzaussage sich als Orakel zeigt.

Viertens ist schließlich die Auslegung der Integrationsermächtigung des Art. 23 GG durch das BVG im Hinblick auf das Verhältnis zwischen mitgliedstaatlicher und europäischer Gerichtsbarkeit in der Rechtsschutzfrage diskussionsbedürftig. Das Gericht postuliert, dass ,,[m]itgliedstaatlichen Rechtsprechungsorganen mit verfassungsrechtlicher Funktion [...] nicht die Verantwortung für die Grenzen ihrer verfassungsrechtlichen Integrationsermächtigung und die Wahrung der unverfügbaren Verfassungsidentität genommen werden“ kann. ${ }^{200}$ Auch wenn man von dieser Grundlage ausgeht, lässt sich Art. 23 GG in Verbindung mit den Zustimmungsgesetzen zum Primärrecht indes gerade derart verstehen, dass jedenfalls der Rechtsschutz gegen politische und administrative Maßnahmen der Europäischen Union der Gemeinschaftsgerichtsbarkeit zu verbindlicher Letztentscheidung auf der Grundlage des ratifizierten Integrationsprogramms zugewiesen ist. Dies schließt weder eine Anerkennung verfassungsrechtlich zwingender Allgemeininteressen durch den EuGH aus (so schon im Fall ,Omega ${ }^{\text {‘201}}$ ) noch (rechtspolitisch) eine Vertragsänderung im Sinne der Zulassung von Alleingängen analog zu derzeitigen Modellen im EGV (zum Beispiel bei der marktintegrativen Rechtsangleichung ${ }^{202}$ ). Das BVG sieht dies anders. Es will die Endgültigkeit von Entscheidungen des EuGH nur ,grundsätzlich“ anerkennen, ${ }^{203}$ relativiert damit folgerichtig auch den Anwendungsvorrang von Gemeinschaftsrecht ${ }^{204}$ und behält sich in Nachfolge des Maastricht-Urteils die Prüfung vor, ob sich europäische Akte ,unter Wahrung des gemeinschafts- und unionsrechtlichen Subsidiaritätsprinzips in den Grenzen der [...] einge-

196 Vgl. Rdz. 240 des Urteils.

197 Vgl. Rdz. 333 des Urteils.

198 Vgl. Rdz. 334 des Urteils.

199 Vgl. Rdz. 238 des Urteils.

200 Vgl. Rdz. 336 des Urteils.

201 EuGH Rs. C-36/02 (Omega), Urteil vom 14.10.2004, Slg. 2004, I-9609.

202 Derzeit Art. 95 Abs. 4 und 5 EGV; projektierter Art. 114 Abs. 4 und 5 AEUV.

203 Vgl. Rdz. 337 des Urteils.

204 Vgl. Rdz. 339 des Urteils. 
räumten Hoheitsrechte halten“. ${ }^{205}$ Dies ist ein starker Anspruch, bei dem, wie Carl Otto Lenz betont, das BVG ,,bei allem Bemühen um Objektivität nicht neutral ist“, weil es um seine eigenen Kompetenzen geht. ${ }^{206}$ Dass eine derartige Kontrollaufgabe dem BVG zukommt, ist eine Selbstpositionierung im komplexen europäischen und deutschen Gewaltenteilungssystem. Zwar beseitigt das Urteil ein bei deutschen Instanzfachgerichten vereinzelt aufgekommenes Missverständnis des Maastricht-Urteils über die Zuständigkeit zur Verwerfung eines Sekundärrechtsaktes, ${ }^{207}$ indem es klarstellt, dass das BVG innerhalb der nationalen Gerichtsbarkeit nur sich selbst die Feststellung eines ,Ultra-vires'-Handelns und einer Verletzung der Verfassungsidentität einräumt. ${ }^{208}$ Diese Aussage könnte Instanzgerichte erneut zu Fehlschlüssen verleiten. Denn nach der Entscheidung des EuGH ,Foto-Frost ‘ ist jedes mitgliedstaatliche Gericht berechtigt, sich die Frage der Gültigkeit eines Sekundärrechtsakts selbst zu stellen, und verpflichtet, bei nicht überwindbaren Zweifeln die Gültigkeitsfrage dem EuGH vorzulegen. ${ }^{209}$ Zwar ist es ein Faktum, dass die letztverbindliche Auslegung des Art. 23 GG beim BVG liegt. Das BVG kann daher, wie vorliegend geschehen, seine eigene Rolle im Gang der europäischen Integration verfassungsrechtlich definieren $^{210}$ und ausweiten. Aber die auch das BVG treffende europarechtliche Vorlagepflicht des BVG zum EuGH aus Art. 234 Abs. 3 EGV, die es mit keinem Wort erwähnt, lässt sich dadurch nicht wegzaubern (näher dazu unten bei den Konsequenzen).

Ungeachtet dieser Frage bleibt zum Schutz eines Mitgliedstaats möglicherweise der (freilich vage) völkerrechtliche Gesichtspunkt der ,clausula rebus sic stantibus ‘ valide. ${ }^{211}$ Dass dies auch in gleicher Weise für den vom BVG sehr allgemein beigezogenen, ordre public'Vorbehalt zu gelten hat, ${ }^{212}$ ist indes zweifelhaft. Mit intellektueller Eleganz, aber schiefer Parallele zu einer nicht supranationalen Organisation beruft sich das Urteil auf das Selbstverständnis des EuGH in dessen Urteil ,Kadi ', 213 in dem dieser grundlegende Rechtsprinzipien des Gemeinschaftsrechts gegen eine Resolution des Sicherheitsrats der Vereinten Nationen durchgreifen ließ. ${ }^{214}$ Hält man insoweit aber eine Funktion des BVG für denkbar, bedürfte dies jedoch, wie schon angesprochen, gemeinschaftsrechtlicher Einbettung und Absegnung: konzeptionell analog zum Modell der immanenten Beschränkung der transnationalen Marktgrundfreiheiten aus zwingenden einzelstaatlichen Allgemeininteressen, ${ }^{215}$ die beispielsweise im ,Omega'-Fall (Vertrieb eines britischen virtuellen Tötungsspiels) zur Rechtfertigung einer Beschränkung der Dienstleistungsfreiheit zum Schutz der Menschenwürde in Deutschland führte. ${ }^{216}$ Der EuGH könnte dieses Modell der Immanenzschranken (mit der damit verbundenen, von ihm kontrollierten Möglichkeit für die ausnahmsweise einzelstaatliche Nichtanwendbarkeit einer sekundärrechtlichen Norm) über die Berücksichti-

205 Vgl. Rdz. 240 des Urteils.

206 Lenz: Ausbrechender Rechtsakt, 2009.

207 Seinerzeit berichtet von Günter Hirsch.

208 Vgl. Rdz. 241 des Urteils.

209 EuGH Rs. 314/85 (Foto-Frost/Hauptzollamt Lübeck-Ost), Urteil vom 22.10.1987, Slg. 1987, 4199.

210 Vgl. Rdz. 336, 343 des Urteils.

211 Zum Problem der Berufung auf Art. 62 Wiener Übereinkommen vom 23. Mai 1969 über das Recht der Verträge (BGB1. 1985 II S. 926) vgl. z.B. Rudolf Streinz, in: Rudolf Streinz (Hrsg.): EUV/EGV. Kommentar, München 2003, Art. 49 EUV Rdz. 17.

212 Vgl. Rdz. 340 des Urteils.

213 Vgl. EuGH verb. Rs. C-402/05 P und C-415/05 P (Kadi und Al Barakaat), Urteil vom 03.09.2008, abgedruckt in: Europarecht 1/2009, S. 80-113.

214 Vgl. Rdz. 340 des Urteils.

215 Vgl. ,Cassis'-Rechtsprechung: EuGH Rs. 120/78 (Rewe/Bundesmonopolverwaltung für Branntwein), Urteil vom 20.02.1979, Slg. 1979, 649.

216 EuGH Rs. C-36/02 (Omega), Urteil vom 14.10.2004, Slg. 2004, I-9609. 
gung substanziierter Einzelgesichtpunkte der „Verfassungsidentität“ eröffnen und damit das vom BVG aufgebaute Konfliktpotenzial unionsrechtlich entspannen.

\section{Integrationspolitische Konsequenzen: Einschränkungen deutscher und europäischer Supranationalitätspolitik?}

Das Lissabon-Urteil wirft für die integrationspolitischen Konsequenzen insbesondere die Frage auf, ob die deutsche und europäische Supranationalitätspolitik eingeschränkt wird. Dies führt zu einem nicht geringen Teil in das Reich von Spekulationen. Immerhin lässt sich aber ein Vorgabenfächer des BVG aufzeigen, aufgrund dessen speziell die Fragen nach Schranken der supranationalen europäischen Rechtsgemeinschaft und nach der Einschränkung der integrationspolitischen Handlungsdynamik behandelt werden können.

Der dreigliedrige Vorgabenfächer des BVG für die Integrationspolitik, insbesondere die Supranationalitätspolitik

Dem Urteil lässt sich ein dreigliedriger Fächer von Vorgaben für die Integrationspolitik, insbesondere die Supranationalitätspolitik, entnehmen.

Inhaltliche verfassungsrechtliche Schranken für die Sekundärrechtspolitik der EU nach dem Inkrafttreten des $V v L$ : Zuallererst fallen inhaltliche verfassungsrechtliche Schranken für die Sekundärrechtspolitik der Europäischen Union ins Auge. Sie betreffen zum einen bestimmte sachgebietliche Erweiterungen der EU-Politikkompetenzen durch den VvL und zum anderen als allgemeine Querschnittsschranken alle Politiken.

Die Verfassungsgemäßheit bestimmter Kompetenzerweiterungen des VvL, insbesondere im Bereich der derzeitigen zweiten und dritten Säule der Europäischen Union, nämlich im sogenannten „Raum der Freiheit, der Sicherheit und des Rechts“217 und in der Sicherheitsund Verteidigungspolitik, sowie in einigen anderen Politikbereichen versieht das BVG mit dem Caveat (Vorbehalt) der ,gebotenen Auslegung“. Dieses umfasst unter anderem im Bereich der strafjustiziellen Zusammenarbeit ${ }^{218}$ das Gebot der ,strikt[en] [...] keinesfalls extensiv[en]“ Auslegung der Kompetenzen ${ }^{219}$ und der (nachvollziehbaren) „engen“ Auslegung bei Entscheidungen zur gegenseitigen Anerkennung von Gerichtsentscheidungen ${ }^{220}$ sowie $^{2}$ die Aufrechterhaltung des Schuldprinzips; ${ }^{221}$ im Bereich der ziviljustiziellen Zusammenarbeit $^{222}$ die Unberührtheit der (ohnehin kaum integrationsverdächtigen) staatlichen Zuständigkeiten für die Organisation des Gerichtswesens sowie der Garantien des effektiven Rechtsschutzes und des Justizgewährungsanspruchs; ${ }^{223}$ im Bereich der Gemeinsamen Handelspolitik die aus dem staatlichen Souveränitätsmodell des BVG abgeleitete, jedenfalls formelle Beibehaltung der Mitgliedschaft der Mitgliedstaaten der Europäischen Union in der Welthandelsorganisation ${ }^{224}$ sowie das Gebot einer engen Auslegung der ausschließlichen

217 Dazu insgesamt Peter-Christian Müller-Graff: Der Raum der Freiheit, der Sicherheit und des Rechts in der Lissabonner Reform, in: Europarecht Beiheft 1/2009, S.105ff.

218 Projektierte Artt. 82ff. AEUV.

219 Vgl. Rdz. 358, 363 des Urteils.

220 Vgl. Rdz. 360 des Urteils. Zum ambitiösen Charakter des Prinzips der gegenseitigen Anerkennung im Strafrecht vgl. Peter-Christian Müller-Graff: Der „Raum der Freiheit, der Sicherheit und des Rechts“ im neuen Verfassungsvertrag für Europa-Neuerungen und Notwendigkeit seiner Rekonstruktion, in: Charlotte Gaitanides/ Stefan Kadelbach/Gil Carlos/Rodriguez Iglesias (Hrsg.): Europa und seine Verfassung. Festschrift für Manfred Zuleeg, Baden-Baden 2005, S. 605-623, hier S. 615.

221 Vgl. Rdz. 364 des Urteils.

222 Projektierter Art. 81 AEUV.

223 Vgl. Rdz. 368 des Urteils.

224 Vgl. Rdz. 375 des Urteils. 
Zuständigkeit für ausländische Direktinvestitionen (Beschränkung auf Kontrollerwerb). ${ }^{225}$ Das BVG entwickelt sein Auslegungsverständnis auch zu den Bestimmungen über die Sicherheits- und Verteidigungspolitik (namentlich Parlamentsvorbehalt für den Einsatz der Bundeswehr) ${ }^{226}$ und über die vom VvL freilich nur marginal berührte Sozialpolitik. ${ }^{227}$ Der damit vom BVG ausgesprochene Verständnisvorbehalt für die Konformität des VvL mit dem GG ist zwar nicht verbindlich für die anderen 26 Mitgliedstaaten und die europäischen Organe, signalisiert aber ein Risikopotenzial für die Befolgung einschlägigen Sekundärrechts in Deutschland und damit für die europäische Rechtsgemeinschaft. Er kann dadurch zu einer vorgelagerten Hemmschwelle für Rechtsetzungsinitiativen werden.

Daneben stehen die allgemeinen Querschnittsschranken für alle Politiken. Zu den in der

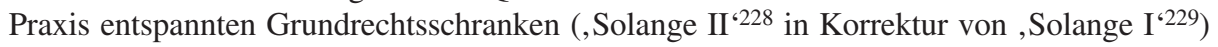
und zur ,Ultra-vires'-Schranke (,Ausbrechender Rechtsakt““ im Maastricht-Urteil ${ }^{230}$ ) gesellt sich nun die Schranke der ,Verfassungsidentität“. ${ }^{231}$ Dieser Gesichtspunkt lässt sich potenziell breitflächig (gegen Richtlinien und Verordnungen) in Stellung bringen; ob mit Erfolg, ist freilich eine andere Frage.

Verfassungsrechtliche Verfahrensvoraussetzungen der deutschen Mitwirkung bei der Nutzung ,dynamischer Entwicklungsbestimmungen ' des VvL: Eine zweite Gruppe verfassungsrechtlicher Vorgaben betrifft speziell die Verfahrensvoraussetzungen der deutschen Mitwirkung an den Entscheidungen der vom VvL eingeführten oder veränderten Fälle ,dynamische[r] Vertragsentwicklung“. Hierbei geht es insbesondere um die vereinfachten Änderungsverfahren, die Brückenklauseln und die Flexibilitätsklausel sowie das Notbremseverfahren. Das BVG begründet hierfür aus dem Prinzip der parlamentarischen Integrationsverantwortung kleinteilig verfassungsrechtliche Erfordernisse der Mitwirkung von Bundestag und Bundesrat, die zwischenzeitlich in den Entwurf des Integrationsverantwortungsgesetzes (IntVG) ${ }^{232}$ getreulich übernommen sind. ${ }^{233}$ Damit werden die betroffenen Entscheidungen, nicht aber auch der Alltag europäischer Rechtsetzung, jeweils einer parlamentarischen Beschlussfassung zugewiesen. ${ }^{234}$ Dies eröffnet einerseits eine Chance zu einer in der nationalen Öffentlichkeit geführten parlamentarischen Debatte, die vom BVG zu Recht als demokratisch primär bedeutsam angesehen wird. Es trägt damit der allgemeinen Einsicht Rechnung, dass die Gegenstände europäischer Integration nicht nur Außenpolitik, sondern auch Innenpolitik sind, wobei allerdings auch zu beachten bleibt, dass sie nicht nur Innenpolitik, sondern auch Außenpolitik sind. Andererseits bringt das BVG dadurch auch hier, ohne es anzusprechen, sich selbst ins Spiel: als Kontrolleur über die VerfassungsmäBigkeit entsprechender Beschlüsse.

225 Vgl. Rdz. 379 des Urteils.

226 Vgl. Rdz. 381ff. des Urteils.

227 Vgl. Rdz. 392ff. des Urteils.

228 BVerfGE 37, 271.

229 BVerfGE 73, 339.

230 BVerfGE 89, 155.

231 Rdz. 218 des Urteils.

232 Gesetz über die Wahrnehmung der Integrationsverantwortung des Bundestages und des Bundesrates in Angelegenheiten der Europäischen Union (Integrationsverantwortungsgesetz - IntVG), in: Art. 1 Entwurf eines Gesetzes über die Ausweitung und Stärkung der Rechte des Bundestages und des Bundesrates in Angelegenheiten der Europäischen Union, in: Bundestagsdrucksache 16/13923 vom 21.08.2009, S. 3-5.

233 Vgl. dazu Peter-Christian Müller-Graff zitiert in Nicole Diekmann: Ein Vetorecht für „16 Zwerge“?, in: tagesschau.de, 26.08.2009, abrufbar unter: http://www.tagesschau.de/inland/lissabon108.html (letzter Zugriff: 09.09.2009).

234 Vgl. Rdz. 406ff. des Urteils. Für den Alltag europäischer Rechtsetzung gelten demgegenüber die Neuerungen des VvL (namentlich das Frühwarnverfahren seitens der nationalen Parlamente) sowie in Deutschland die Vorgaben des Art. 23 Abs. 2ff. GG und die Regeln des EUZBBG und des EUZBLG. 
Erstens bindet das Urteil das Inkrafttreten primärrechtlicher materieller Änderungen im vereinfachten Verfahren ${ }^{235}$ an den Erlass eines Gesetzes nach Art. 23 Abs. 1 S. 2 GG, ${ }^{236}$ obwohl vom VvL hierbei eine Zuständigkeitsausdehnung ausdrücklich ausgeschlossen ist. ${ }^{237}$ Es erstreckt dieses Erfordernis auf strukturell entsprechende Änderungsvorschriften. ${ }^{238}$

Zweitens wird für das allgemeine Brückenverfahren der Entscheidungsregeln (Übergang zur Mehrheitsentscheidung im Rat und/oder zum ordentlichen Gesetzgebungsverfahren) ${ }^{239}$ und für die spezielle Brückenklausel im Familienrecht ${ }^{240}$ das vom VvL vorgesehene Ablehnungsrecht jedes nationalen Parlaments nicht als ausreichendes Äquivalent zum Ratifikationsvorbehalt des ordentlichen und des vereinfachten Änderungsverfahrens bewertet und daher das Erfordernis der gesetzlichen Ermächtigung des deutschen Regierungsvertreters zur Zustimmung festgelegt. ${ }^{241}$ Damit wird der Fall der Enthaltung nicht erfasst, ${ }^{242}$ doch legt der Entwurf des IntVG im Falle eines fehlenden Gesetzes die Ablehnungspflicht des deutschen Vertreters im Europäischen Rat fest. ${ }^{243}$ Zugleich macht das Urteil für die speziellen Brückenklauseln ${ }^{244}$ die Erteilung der Zustimmung des Bundestages und gegebenenfalls des Bundesrates zur Voraussetzung für ein positives Votum des deutschen Regierungsvertreters im Rat, wobei das Schweigen von Bundestag/Bundesrat nicht als Zustimmung gewertet werden darf. ${ }^{245}$ Dem dagegen bereits vereinzelt erhobenen Vorwurf unnötigen Misstrauens in die politische Selbstgestaltung im politischen Dreieck von Bundesregierung, Bundestag und Bundesrat lässt sich entgegenhalten, dass in dieser parlamentarischen Rückbindung neuer Integrationsschritte eine systemstimmige Parallele zum parlamentarischen Wesentlichkeitsvorbehalt in innerstaatlichen Angelegenheiten ${ }^{246}$ erkennbar ist und dass überdies die Stärkung der nationalen Parlamente auf der Linie des $\mathrm{VvL}^{247}$ und zugleich im Interesse der Europäischen Union an der darin liegenden Mitwirkungsverantwortung liegt.

Schließlich enthält das Urteil aber auch eine spektakuläre ,Neubewertung ' der sogenannten Abrundungs- oder Flexibilitätsklausel. ${ }^{248}$ Deren quantitative Nutzung war in der Vergangenheit nicht unbedeutsam, ${ }^{249}$ ist aber wegen der primärrechtlich aufgewachsenen speziellen Einzelermächtigungen in ihrer Bedeutung abgesunken. Das BVG gibt vor, die Neubewertung wegen deren textlicher Änderung durch den VvL vornehmen zu müssen. ${ }^{250}$ Die darin liegende Annahme, dass diese Klausel erst durch den VvL für Politikziele auch außerhalb der Marktintegration in Anspruch genommen werden kann, übernimmt im europarechtli-

235 Projektierter Art. 48 Abs. 6 EUV-Liss.

236 Vgl. Rdz. 412 des Urteils.

237 Projektierter Art. 48 Abs. 6 UAbs. 3 EUV-Liss.

238 Vgl. Rdz. 412 des Urteils.

239 Projektierter Art. 48 Abs. 7 EUV-Liss.

240 Projektierter Art. 81 Abs. 3 AEUV.

241 Vgl. Rdz. 319, 366, 414 des Urteils.

242 Vgl. projektierter Art. 235 Abs. 1 UAbs. 3 AEUV: „Die Stimmenthaltung von anwesenden oder vertretenen Mitgliedern steht dem Zustandekommen von Beschlüssen des Europäischen Rates, zu denen Einstimmigkeit erforderlich ist, nicht entgegen."

243 Entwurf des $§ 4$ Abs. $1 \mathrm{~S}$. 2 IntVG.

244 Vgl. projektierte Art. 48 Abs. 7 EUV-Liss., Art. 81 Abs. 3 UAbs. 3 AEUV; ferner projektierte Art. 31 Abs. 3 EUV-Liss., Artt. 153 Abs. 2 UAbs. 4, 192 Abs. 2 UAbs. 2, 312 Abs. 2 UAbs. 2, 333 Abs. 1 und 2 AEUV.

245 Vgl. Rdz. 320 des Urteils.

246 Vgl. etwa BVerfGE 49, 89, 126 und BVerfGE 77, 170, 230f.; dazu Michael Sachs, in: Michael Sachs (Hrsg.): Grundgesetz. Kommentar, 3. Aufl., München 2003, Art. 20 Rdz. 116f.

247 Vgl. Protokoll über die Rolle der nationalen Parlamente in der Europäischen Union, in: Amtsblatt der EU, Nr. C 306 vom 17. Dezember 2007, S. 148-150; Protokoll über die Anwendung der Grundsätze der Subsidiarität und der Verhältnismäßigkeit, in: Amtsblatt der EU, Nr. C 306 vom 17. Dezember 2007, S. 150-152.

248 Projektierter Art. 352 AEUV; derzeit Art. 308 EGV.

249 Vgl. als Statistik Becker/Maurer: Deutsche Integrationsbremsen, 2009.

250 Vgl. Rdz. 327 des Urteils. 
chen Streit um die Tragweite des derzeitigen Art. 308 EGV 251 einseitig die eher minoritäre Meinung und widerspricht der Praxis. ${ }^{252}$ Das BVG untersagt ab Inkrafttreten des VvL dem deutschen Vertreter im Rat die förmliche Zustimmung zu einem auf die Abrundungsklausel gestützten Vorschlag der Kommission, solange diese Inanspruchnahme nicht durch ein Gesetz von Bundestag und Bundesrat gemäß Art. 23 GG ,ratifiziert“ ist. ${ }^{253}$ Sachgegenständlich erfasst dies künftig nicht nur Neuerungen der Europäischen Fusionskontrolle oder die Schaffung europäischer Gesellschaftsformen, sondern auch nachrangige organisationelle Fragen (zum Beispiel die Schaffung von Agenturen), denen gegenüber ein Gesetzesvorbehalt (regelmäßig wohl ohne das Erfordernis einer verfassungsändernden Mehrheit) unverhältnismäBig wirkt. Da die Enthaltung eines Mitglieds im Rat der Einstimmigkeit nicht entgegensteht, ${ }^{254}$ wird das vom BVG verfolgte Ziel jedoch nur erreicht, wenn der Regierungsvertreter bei fehlender gesetzlicher Grundlage zur Ablehnung im Rat verpflichtet wird. ${ }^{255}$ Der Entwurf des IntVG zieht diese Konsequenz.

Das BVG unterstellt des Weiteren das Handeln der Bundesregierung im Rat im Notbremseverfahren den Weisungen des Bundestages und gegebenenfalls des Bundesrats ${ }^{256}$ und stellt in der strafjustiziellen Zusammenarbeit bestimmte Beschlussfälle unter Gesetzesvorbehalt. ${ }^{257}$

Verfassungsrechtliche Schranken deutscher Primärrechtspolitik: Schließlich substanziiert das Urteil, wie aufgezeigt, mit den Kategorien der „Verfassungsidentität“ und der ,,souveränen Staatlichkeit" neu formulierte verfassungsrechtliche Schranken auch für die deutsche Primärrechtspolitik. So wird, wie gezeigt, insbesondere das Modell des Eintritts in einen ,europäischen Bundesstaat“ aus dem Grundgesetz verwiesen und dem Erfordernis einer neuen Verfassungsgebung (Art. 146 GG) unterstellt. Zweifelhaft ist, ob primärrechtspolitisch ausgreifende Konzepte einer europäischen Armee, Sozialversicherung oder Besteuerungskompetenz mit der „Verfassungsidentität“ vereinbar sind. 258

\section{Abschwächung der supranationalen europäischen Rechtsgemeinschaft?}

Soweit sich die Integrationspolitik europarechtlicher Mittel bedient, wird die Frage aktuell, ob das Lissabon-Urteil eine Bahn vorzeichnet, die das Modell der verbindlichen Supranationalität zugunsten des in seiner Verbindlichkeit schwächeren klassischen Völkerrechts favorisiert.

Der Aufwuchs allgemeiner völkerrechtlicher Topoi in der Argumentation des LissabonUrteils: Im Argumentationsgang des Urteils fällt ein Aufwuchs allgemeiner völkerrechtlicher Topoi auf: die argumentative Gleichstellung der Europäischen Gemeinschaft/Europäischen Union mit klassischen internationalen Organisationen; 259 die Begründung eines (mit dem derzeitigen Unionsrecht nicht vereinbaren) Austrittsrechts aus allgemeinem Völkerrecht; ${ }^{260}$ die Argumentationsparallele zwischen VN-Sicherheitsrat und Europäischer Gemeinschaft/Europäischer Union; ${ }^{261}$ die massive Relativierung der Kernelemente rechtlicher

251 Vgl. ausführlich Ivo E. Schwartz, in: Hans von der Groeben/Jürgen Schwarze (Hrsg.): EUV/EGV. Kommentar, 6. Aufl., München 2003, Art. 308 EGV Rdz. 83ff., 147ff., 163ff.

252 Ebenda, Art. 308 EGV Rdz. 160.

253 Vgl. Rdz. 327, 417 des Urteils.

254 Projektierter Art. 238 Abs. 4 AEUV; derzeit Art. 205 Abs. 3 EGV.

255 So jetzt auch der Entwurf von $\S 8$ IntVG.

256 Vgl. Rdz. 365, 400 des Urteils. Dies betrifft die projektierten Artt. 48 Abs. 2, 82 Abs. 3, 83 Abs. 3 AEUV.

257 Rdz. 419 des Urteils.

258 Skeptisch Becker/Maurer: Deutsche Integrationsbremsen, 2009, S. 3.

259 Vgl. Rdz. 229, 237 des Urteils.

260 Vgl. Rdz. 233, 264 des Urteils.

261 Vgl. Rdz. 340 des Urteils. 
Supranationalität (hinsichtlich unmittelbarer Anwendbarkeit ${ }^{262}$ und Anwendungsvorrang ${ }^{263}$ mit vom BVG nicht angesprochenen, aber naheliegenden Folgewirkungen auf das Gebot der richtlinienkonformen Auslegung nationalen Rechts ${ }^{264}$ und die Staatshaftung bei qualifizierter Verletzung supranationalen Rechts ${ }^{265}$ ); die Bemühung des , ordre public'-Gedankens, ${ }^{266}$ wie er etwa dem internationalen Privatrecht im Verhältnis zwischen verschiedenen gleichrangigen Rechtsordnungen zu eigen ist; ${ }^{267}$ und, nicht zuletzt, die Leugnung des Letztentscheidungsrechts des EuGH in der Auslegung von Gemeinschaftsrecht. ${ }^{268}$ Dies alles sind Kategorien, die der Bedeutung und Besonderheit der europäischen Integration, die ihr das GG in Präambel und Art. 23 GG beimisst, nicht gerecht werden. Sie sind geeignet, die Grundfesten der großen innovativen europäischen Rechtsgemeinschaft seit 1952 zu schwächen und den Weg in die demokratisch weit weniger kontrollierten Formen intergouvernementaler Zusammenarbeit zu stärken (zum Beispiel als Menetekel: ,Bologna').

Gefahren für die supranationale Rechtsgemeinschaft: Insbesondere eröffnet das Lissabon-Urteil mit der einem Truppenaufzug ähnelnden breitflächig erörterten Möglichkeit, dass ein europäischer Rechtsakt in der Bundesrepublik unanwendbar sein könne, für die europäische supranationale Rechtsgemeinschaft eine Büchse der Pandora. Die eigenständige Erklärung der Nichtanwendbarkeit wäre nicht nur ein gravierender Einschnitt in das von allen getragene Idealbild der europäischen Rechtsgemeinschaft, wie sie von Walter Hallstein als ein Fundament für das dauerhafte Gelingen der Europäischen Gemeinschaft hervorgehoben wurde. ${ }^{269}$ Eine derartige Relativierung wäre insbesondere auch deshalb gravierend, weil die Nachahmungsgefahr bestünde, dass der Anspruch des BVG als des Verfassungsgerichts des größten Mitgliedstaats auch von jedem vergleichbaren Gericht der anderen 26 Mitgliedstaaten nach jeweils eigenen Maßstäben erhoben werden könnte. Stellt aber jedes dieser Gerichte die Anwendbarkeit von Rechtsakten der Europäischen Union in seinem Jurisdiktionsgebiet unter jeweils spezifische nationale Verfassungsvorbehalte, könnte darüber hinaus auch die Bereitschaft der jeweiligen staatlichen, politischen und administrativen Organe gröBer werden, den Spielraum für Vertragsverletzungen stärker auszutesten. Damit würden aber für die politische und administrative Praxis in den betroffenen Sachbereichen (bis hin zum Binnenmarkt) auch die Gebote der unmittelbaren Anwendbarkeit und der richtlinienkonformen Auslegung nationalen Rechts, des Anwendungsvorrangs des europäischen Rechts und der Schadensersatzhaftung wegen (qualifizierter) Verletzung des Unionsrechts geschwächt. Letztlich münden diese Probleme immer wieder in die Frage, in welchem Maße es Aufgabe einer einzelnen mitgliedstaatlichen Judikative sein kann, im eigenen Hoheitsgebiet die Entscheidungen europäischer Hoheitsträger zu verwerfen. Die maßgebliche gerichtliche Kontrolle sollte im Interesse der Autorität der europäischen Rechtsgemeinschaft von einem Unionsgericht wahrgenommen werden. Die Bereitschaft, diesem vom Primärrecht vorge-

262 Grundlegend EuGH Rs. 26/62 (van Gend \& Loos), Urteil vom 05.02.1963, Slg. 1963, 3.

263 Grundlegend EuGH Rs. 6/64 (Costa/ENEL), Urteil vom 15.07.1964, Slg. 1964, 1253.

264 Grundlegend EuGH Rs. 14/83 (von Colson und Kamann), Urteil vom 10.04.1984, Slg. 1984, 1891.

265 EuGH verb. Rs. C-6/90 und C-9/90 (Francovich), Urteil vom 19.11.1991, Slg. 1991, I-5357; EuGH verb. Rs. C-178/94, C-179/94, C-188/94, C-189/94 und C-190/94 (Dillenkofer u.a.), Urteil vom 08.10.1996, Slg. 1996, I-4845.

266 Vgl. Rdz. 340 des Urteils.

267 Vgl. etwa Art. 6 Einführungsgesetz zum Bürgerlichen Gesetzbuche in der Fassung der Bekanntmachung vom 21. September 1994 (BGB1. 1994 I S. 2494) zuletzt geändert durch Art. 4 Abs. 9 des Gesetzes vom 11. August 2009 (BGB1. 2009 I S. 2713, 2721).

268 Vgl. Rdz. 340 des Urteils.

269 Vgl. Walter Hallstein: Die Europäische Gemeinschaft, 5. Aufl., Düsseldorf/Wien 1979, S. $51 \mathrm{ff}$. 
zeichneten Weg in der Praxis zu folgen, hängt freilich gerade auch von dem Maß des Vertrauens ab, das der EuGH in seiner Kontrollleistung in den Mitgliedstaaten genießt.

Die Bewältigung der Selbstpositionierung des Bundesverfassungsgerichts im System der deutschen und europäischen Gewaltenteilung: Allerdings wird die mit der Verfassungsauslegung einhergehende europapolitische Selbstpositionierung des BVG im System der deutschen und europäischen Gewaltenteilung zu einer Selbstherausforderung des BVG im Lichte seiner eigenen Integrationsverantwortung führen, die ihm noch zu schaffen machen wird. Denn es wird im Gefolge seiner großzügig wirkenden Einladung zu Verfassungsbeschwerden gegen europäische Rechtsakte zuallererst Mechanismen zu entwickeln haben, um die zu erwartenden Versuche seiner allfälligen politischen Instrumentalisierung einzudämmen. Es wird darüber hinaus wegen der auch das BVG aus Präambel und Art. 23 GG treffenden „Integrationsverantwortung“ für die zügige und sachkundige Behandlung der bei ihm anhängigen Verfahren zu sorgen und der Bundesrepublik europapolitischen Gestaltungsspielraum zu sichern haben. Und es wird mit seiner Selbstpositionierung vor allem zu beachten haben, dass Fragen der Auslegung insbesondere des AEUV und der Gültigkeit oder Auslegung europäischen Sekundärrechts das BVG zu einem vorlagepflichtigen Gericht gegenüber dem EuGH machen. ${ }^{270}$ Denn wenn es bei der verfassungsrechtlichen Prüfung eines europäischen Rechtsaktes im Rahmen des AEUV inzident auf die Auslegung primäroder sekundärrechtlicher Ermächtigungsnormen oder materiellrechtlicher Normen ankommt, wird offensichtlich im Sinne der Vorlagevorschriften des AEUV die Auslegung des Vertrages oder die Gültigkeit oder Auslegung der Handlungen der Organe der Europäischen Union für den Erlass der Entscheidung des BVG erforderlich. ${ }^{271}$ Der Einwand, es werde nur das Zustimmungsgesetz ausgelegt, aber nicht Gemeinschaftsrecht, ist rabulistisch. Denn das Zustimmungsgesetz ist allein die legitimatorische Hülle für Bestimmungen, die in Gegenseitigkeit vereinbart wurden und deren verbindliche Auslegung im Interesse wechselseitig verlässlicher Verbindlichkeit der Gemeinschaftsgerichtsbarkeit zugewiesen ist ${ }^{272}$ (derzeit Artt. 220ff. EGV). Es ist die Lösung, die von deutscher Seite seit 1952 beziehungsweise 1957 durch die einschlägigen Zustimmungsgesetze ${ }^{273}$ in Verbindung mit den Integrationsklauseln des GG ratifiziert worden ist.

Würde das BVG einen europäischen Rechtsakt wegen Überschreitung des „Integrationsprogramms“ oder wegen Verletzung der „Verfassungsidentität“ von sich aus für unanwendbar in Deutschland erklären, ohne sich hierbei auf eine Entscheidung des EuGH berufen zu können, träfe es dasselbe Verdikt, das es selbst in der Rechtssache ,Kloppenburg ' gegenüber dem Bundesfinanzhof, der die unmittelbare Anwendung einer Richtlinienbestimmung entgegen der Rechtsprechung des EuGH verweigert hatte, ausgesprochen hat: ${ }^{274}$ Die Nichtvorlage der Gültigkeits- und/oder der Auslegungsfrage wäre in der Eigendiktion des BVG eine „objektiv willkürliche“ Verletzung des Rechts auf den gesetzlichen Richter, die im Widerspruch zu Art. 101 Abs. 1 GG stünde. Würde das BVG entweder ohne Vorlage einen europäischen Rechtsakt in Deutschland für unanwendbar erklären oder nach Bestätigung der Gültigkeit durch den EuGH davon ohne erneute Vorlage abweichen, müsste die Frage der europarechtlichen Zulässigkeit eines derartigen Verhaltens mittels eines von der Kommis-

270 Derzeit Art. 234 Abs. 3 EGV; projektiert Art. 267 Abs. 3 AEUV.

271 Dis gilt im Rahmen der projektierten Art. 267 Abs.1 lit. a AEUV (,,der Verträge“) und Art. 19 Abs. 3 lit. b EUV-Liss. (einschränkend zum EUV-Liss. „,nach Maßgabe der Verträge“).

272 Derzeit Artt. 220ff. EGV, 46 EUV; projektierte Art. 19 EUV-Liss., Artt. 251ff. AEUV.

273 Nachweis der Zustimmungsgesetze in Peter-Christian Müller-Graff: Primärrechtliche Entwicklungsschritte der Gemeinschaftsintegration zu einem transnationalen Gemeinwesen, in: integration 4/2007, S. 407-421.

274 BVerfGE 75, 223ff. 
sion einzuleitenden Vertragsverletzungsverfahrens ${ }^{275}$ durch den EuGH geklärt werden. Dieser hätte spätestens dann die Gelegenheit, die Rechtfertigung eines nationalen Alleingangs von einer Sekundärrechtsbestimmung aus zwingenden verfassungsrechtlichen Gründen zu erwägen. Schon die bloße Einführung eines spezifischen innerstaatlichen Überprüfungsverfahrens vor dem BVG, wie es das Urteil andeutungsweise thematisiert, ${ }^{276}$ wäre im Falle der Zuerkennung einer eigenständigen Kompetenz des BVG zum Ausspruch der Nichtanwendbarkeit einer unionsrechtlichen Norm ein Gefährdungsfanal der supranationalen Rechtsgemeinschaft. Wollte man aber, um den sehr fragenbehafteten Prüfungsansatz über die Verfassungsbeschwerde und das Wahlrecht zum Bundestag spezialgesetzlich zu überwinden, dem Gedanken eines speziellen innerstaatlichen verfassungsgerichtlichen Prüfungsverfahrens nähertreten, müsste dies mit der Klarstellung der Vorlagepflicht des BVG an den EuGH verbunden werden. ${ }^{277}$ Unabhängig davon verdient die mittlerweile von einer Juristengruppe vorgeschlagene deklaratorische Einfügung der Vorlagepflicht des BVG in das Bundesverfassungsgerichtsgesetz ${ }^{278}$ sorgfältige Prüfung. ${ }^{279}$ Insgesamt ist zu hoffen, dass das BVG seine Selbstherausforderung im Sinne einer wie bisher ${ }^{280}$ im Ergebnis konstruktiven Verbindung von Verfassungsverständnis und europäischer Integration zu meistern verstehen wird, und dass der EuGH eine Rechtsprechungslinie findet, die die hypothetischen Problemlagen des BVG unionsrechtlich einfängt.

\section{Einschränkungen der integrationspolitischen Handlungsdynamik?}

Der Schrankenfächer des BVG und die damit verbundenen Unklarheiten über die Soliditätszukunft der supranationalen Rechtsgemeinschaft führen auf die abschließende Frage, ob daraus Einschränkungen der integrationspolitischen Handlungsdynamik resultieren. Naturgemäß sind Aussagen dazu schon deshalb spekulativ, weil der Integrationspolitik differenzierte Reaktionsoptionen zur Verfügung stehen. ${ }^{281}$

Einschränkungen für die politischen Akteure? Zuallererst stellt sich diese Frage für die Europapolitik der Bundesrepublik. Das Lissabon-Urteil fordert hier keinen Konzeptwechsel in eine timide Selbstbeschränkung. Eine derartige Reaktion, die einem ,Paradigmenwechsel im europapolitischen Selbstverständnis Deutschlands“ gleichkäme, ${ }^{282}$ wäre im Licht der Maxime falsch, dass die europäische Integration ein Essential deutscher Selbstbehauptung in

275 Derzeit Art. 226 EGV; projektiert Art. 258 AEUV.

276 Vgl. Rdz. 241 des Urteils.

277 Fraglich bleibt nach dem Lissabon-Urteil freilich, ob sich das BVG sein aus dem GG abgeleitetes ,Letztentscheidungsrecht" vom einfachen Gesetzgeber, also ohne Verfassungsänderung, entwinden lässt.

278 Vorschlag eines § 13a Bundesverfassungsgerichtsgesetz von Jan Bergmann und Ulrich Karpenstein mit folgender Formulierung: „Ist in einem Verfahren vor dem Bundesverfassungsgericht die Auslegung der vertraglichen Grundlagen der Europäischen Union oder die Gültigkeit und die Auslegung der Handlungen der Organe, Einrichtungen oder sonstigen Stellen der Europäischen Union entscheidungserheblich, ist das Bundesverfassungsgericht zur Vorlage dieser Frage an den Gerichtshof der Europäischen Union verpflichtet“. Jan Bergmann/Ulrich Karpenstein: Identitäts- und Ultra-vires-Kontrolle durch das BVerfG - zur Notwendigkeit einer gesetzlichen Vorlageverpflichtung, eingereichtes Manuskript, abrufbar unter: http://verfassungsblog.de/ _oneclick_uploads/2009/08/zeus-bverfg-vorlagepflicht-bergmann-karpenstein-21-8-2009.pdf (letzter Zugriff: 09.09.2009).

279 Auch insoweit könnte das BVG allerdings dem einfachen Gesetzgeber das Recht absprechen, ihm das aus dem GG abgeleitete „Letztentscheidungsrecht“ zu entziehen.

280 So in seiner restriktiven Haltung zur Zulässigkeit von Vorlagen zur Überprüfung von Sekundärrecht anhand des innerstaatlichen Grundrechtsschutzes BVerfGE 102, 147.

281 Zu den Optionen Becker/Maurer: Deutsche Integrationsbremsen, 2009, S. 7-8; sie nennen unter realistischer Ausklammerung des „Sprung[s] zum europäischen Bundesstaat“ für die deutsche Europapolitik insbesondere die Bremser-Option, das „Herantasten an den Kern der Souveränität“ und die Suche nach intergouvernementalen Lösungen außerhalb der Europäischen Union.

282 So zu Recht Becker/Maurer: Deutsche Integrationsbremsen, 2009, S. 8. 
Europa und in der Globalisierung darstellt und daher aktive Gestaltung der Integration statt furchtsamer Defensive gefordert ist. Angesichts der hohen Abstraktheit und orakelartigen Unschärfe der Schranken des BVG bleibt den politischen Organen der gesamte Entscheidungsspielraum, auf europäischer Ebene auf der Grundlage und im Rahmen des Primärrechts das zu bewegen, was vernünftig erscheint, und notfalls die Aufgabe, das BVG davon zu überzeugen. Eine derart konstruktive Grundhaltung zur europäischen Integration ist vor allem auch den Partnerländern zu kommunizieren.

Eine andere Frage betrifft das Gewaltenteilungsverhältnis von Bundesregierung, Bundestag und Bundesrat/Ländern in europäischen Angelegenheiten. Gewiss entstehen mit der Stärkung von Bundestag und Bundesrat/Ländern potenziell zusätzliche Verhandlungsaufwände, sachfremde Blockademöglichkeiten, Versuchungen zu Kopplungsgeschäften und ähnliches mehr. ${ }^{283}$ Ausschlaggebend sollte dabei jedoch jeweils die Abwägung der Vorteile und Nachteile unter dem entscheidungsleitenden Gesichtspunkt des Integrationsnutzens für die Bundesrepublik sein. Daher steht zu hoffen, dass letztlich sinnfällige Integrationsschritte nicht blockiert werden. Und wenn etwas unterbleibt, das nicht sinnfällig ist, ist es kein Schaden.

Im Übrigen ist schwer abzuschätzen, in welchem Umfang die Gestaltungsbereitschaft und Handlungsfähigkeit von EU-Organen infolge innerstaatlicher Bindungsregeln der Bundesregierung betroffen ist. Das Karlsruher Lissabon-Orakel mag freilich anderen Mitgliedstaaten als willkommener Vorwand dienen, Initiativen zu erschweren oder zu blockieren.

Integrationspolitik in einem verdichteten Rechtskorsett? Kontrollanspruch, Kontrollerweiterung, Auslegungsverständnis und die schleierhafte Unschärfe der hochabstrakten Maßstäbe des BVG können innerhalb der Bundesrepublik, aber über das Vorlageverfahren auch vor dem EuGH zu mehr Verfahren und dadurch auch zu integrationspolitischen Verzögerungen führen. Es dürfte nicht fernliegend sein, auch im Lissabon-Urteil eine von Dieter Grimm für die deutsche Verfassungsgeschichte aufgezeigte Eigenheit zu erkennen, in der dem rechtlichen Prozess mehr Vertrauen entgegengebracht wird als dem politischen Prozess (,Rechtsstaat rangiert vor Demokratie"). ${ }^{284}$ Tatsächlich erfüllt das Recht auch für die europäische Integration die Aufgabe eines unschätzbar wertvollen elementaren Stabilitätsankers verbindlicher Gemeinsamkeit und längerfristiger Erfahrungsvernunft in den allfälligen tagespolitischen Willenswellenschlägen von 27 Mitgliedstaaten. Allerdings verdankt es diese Leistung seiner besonderen Eigenheit als supranationale Rechtsgemeinschaft, die nationale rechtliche Alleingänge ohne gemeinschaftsrechtliche Absegnung unterbindet. Die Erfahrung mit diesem Rechtsintegrationsmodell trägt die generelle Annahme, dass die Autorität der supranationalen Rechtsgemeinschaft umso höher sein dürfte, je überzeugender supranationales Sekundärrecht inhaltlich diszipliniert wird, ${ }^{285}$ je nachvollziehbar einsichtiger dessen Legitimation ausfällt (hier ist die Einführung der doppelten Mehrheit im Rat ein bahnbrechender Fortschritt) und je sorgfältiger die Kontrolle von Sekundärrecht durch den EuGH ausgeübt wird. ${ }^{286}$

283 Dazu Becker/Maurer: Deutsche Integrationsbremsen, 2009.

284 Dieter Grimm: Der Weg zur Musterverfassung, in: Frankfurter Allgemeine Zeitung. Redaktionsbeilage „60 Jahre Grundgesetz“, 22.05.2009.

$285 \mathrm{Zu}$ den sich mehrenden Problemen und den Konzeptionselementen funktionsadäquater europäischer Rechtsetzung Peter-Christian Müller-Graff: Der Stil der europäischen Rechtsetzung - Befunde, Herausforderungen, Aufgaben -, in: Michael Gruber/Friedrich Harrer (Hrsg.): Europäische Rechtskultur. Symposium für Heinrich Honsell zum 65. Geburtstag, München 2009, S. 1ff.

286 Hier gibt es gewiss Verbesserungsmöglichkeiten. So vermisst man etwa im zweiten Tabakwerbeurteil des EuGH (Rs. C-380/03 (Deutschland/Parlament und Rat), Urteil vom 12.12.2006, Slg. 2006, I-11573) die erforderliche Differenzierung im Blick auf die Lokal- und Regionalzeitungen und eine profunde Verhältnismäßigkeitsprüfung zwischen der Beschränkung des transnationalen Vertriebs meinungstragender Produkte und dem geringen Gewinn an Gesundheitsschutz durch ein Werbeverbot in diesen Produkten. 
Die im Lissabon-Urteil angelegte Stärkung der Rolle des BVG beinhaltet indes nicht notwendig eine Einschränkung integrationspolitischer Konzeptionen. Zwar wird der (ohnehin nicht aktuellen) Vision eines europäischen Bundesstaates (im engführenden Sinne des Modells der deutschen Bundesstaatlichkeit) die Vorbedingung der Schaffung einer neuen Verfassung gestellt. Auch wirken die zugrunde liegenden Denk- und Sprachmuster des Urteils oft eher verteidigend als neuigkeitsoffen (namentlich: durchgängiger Schutztopos in der Argumentation bis hin zu einer einseitigen Sicht auf den Abbau des Einstimmigkeitserfordernisses ${ }^{287}$ ). Gleichwohl verbietet diese Ausrichtung weder das sich seit mehr als einem halben Jahrhundert vollziehende innovative Entdeckungsverfahren neuer Integrationsschritte noch die Verfolgung von Konzepten stärkerer Integration, die sich für alle Mitgliedstaaten als sinnfällig darstellen. Der Vorsitzende des Zweiten Senats des BVG bekundet in Wiederbelebung einer früheren Politikkategorie sogar, dass das Urteil auch einen europäischen Bundesstaat nicht ausschließe. ${ }^{288}$ Und der vergleichsweise weitaus bescheidenere Sprachgebrauch der funktionellen Verfassungskategorie für das geltende Primärrecht wird nicht unterdrückt, wenn das BVG sehr treffend von den Völkern Europas als „Quelle der [die] Gemeinschaftsgewalt [...] konstituierenden europäischen Verfassung im funktionellen Sinne“ spricht ${ }^{289}$ und die ,„Verfassung Europas“, das Völkervertrags- oder Primärrecht“ als ,,abgeleitete Grundordnung“ bezeichnet. ${ }^{290}$

\section{Zusammenfassung}

Zusammenfassend ist festzuhalten: Das Lissabon-Urteil des BVG setzt der grundgesetzlich zulässigen Integrationspolitik inhaltlich überwiegend hochabstrakte, wertungsoffene und daher auslegungsbedürftige Bedingungen und Grenzpunkte, die sinnfälliger Integrationspolitik den Spielraum erhalten. Es bindet die Beteiligung an einem staatsanalog gedachten europäischen Bundesstaat an die Ablösung des GG durch eine neue Verfassung, blockiert damit aber nicht grundsätzlich den föderativartigen Eigenweg der aufgewachsenen Europäischen Gemeinschaft/Europäischen Union. ${ }^{291}$ Es bestimmt prozedural für die innerstaatliche Legitimation zur Mitwirkung an primärrechtsrelevanten Schritten, die im VvL angelegt sind, oder an der Kompetenzabrundung sehr konkrete (bei der Abrundungsklausel wohl überzogene) Erfordernisse. Es bekräftigt judikativ einen Kontrollanspruch des BVG über sekundäres Europarecht, ohne jedoch die Vorlagepflicht zum EuGH zu erwähnen, die auch das BVG trifft, wenn namentlich im Rahmen des AEUV Fragen der Auslegung sowie der Gültigkeit und Auslegung von Sekundärrecht entscheidungserheblich sind. Europarechtlich lassen sich die dazu angedeuteten Gravamina des BVG mit ihrer potenziellen Sprengkraft für die europäische Rechtsgemeinschaft durch eine kluge Rechtsprechung des EuGH auffangen.

287 In Rdz. 317 des Urteils sieht das BVG bei einem derartigen Übergang nur den Aspekt der Einflussabnahme des deutschen Vertreters im Rat, nicht aber die Steigerung der Gestaltungsmöglichkeiten durch Überwindung des Blockadepotenzials jedes einzelnen anderen Mitgliedstaates.

288 So Andreas Voßkuhle auf einer Podiumsdiskussion am 06.07.2009 in Freiburg in Erwiderung der Kritik von Ernst-Wolfgang Böckenförde; zitiert nach Verlag C. H. Beck, beck-aktuell-Redaktion: BVerfG-Richter Voßkuhle: Europäischer Bundesstaat denkbar, Pressemeldung, 07.07.2009.

289 Vgl. Rdz. 231 des Urteils; das Maastricht-Urteil sprach von der „Gemeinschaftsverfassung“ (vgl. BVerfGE 89, 155, 212); zur konzeptionellen Seite der Integrationsverfassung Peter-Christian Müller-Graff: Die Zukunft des europäischen Verfassungstopos und Primärrechts nach der deutschen Ratspräsidentschaft, in: integration 3/2007, S. 223-237, hier S. 235ff.

290 Rdz. 231 des Urteils.

291 Vgl. zu diesem Eigenweg in ein (abgeleitetes) transnationales Gemeinwesen mit föderativen Zügen MüllerGraff: Primärrechtliche Entwicklungsschritte, 2007. 
Konzeptionell zeigt das Urteil, dass das Spannungsverhältnis zwischen dem historisch neuartigen Entdeckungsverfahren der supranationalen europäischen Integration und dem klassischen staatlichen Souveränitätsdenken, wenig verwunderlich, unverändert Mühe bereitet. Dieses Spannungsverhältnis ist nur aus einem Verständnis zu bewältigen, das den Gesichtspunkt der staatlichen Selbstbehauptung mittels der europäischen Integration einbezieht. Offenkundig ist es unmöglich, sich in eine supranationale Gemeinschaft integrieren und zugleich die bestehende Hoheitsordnung unverändert aufrechterhalten zu wollen. Nationale Souveränität und europäische Hoheitsausübung sind daher nicht einseitig als Gegensatz oder Konkurrenzverhältnis, sondern in einem Ergänzungsverhältnis zu konzipieren. ${ }^{292}$ Europäische Hoheitsausübung steht weder beziehungslos noch generell konträr zu staatlicher Souveränität. Vielmehr vollzieht sie sich im Kernbereich der Rechtspolitik nicht ohne gleichzeitige staatliche Hoheitsausübung. Am deutlichsten wird dies im Entscheidungsverfahren des auch nach dem VvL politisch mächtigsten Organs der Europäischen Union, dem Rat, für dessen demokratische Legitimationsvermittlung daher die Einführung der doppelten Mehrheit durch den VvL ein herausragender Fortschritt ist. Erforderlich ist im Rahmen der europäischen Integration ein verfassungsrechtlich offener Souveränitätsbegriff, der die transnationale Verflechtung einbezieht, mithin die primären Verbandskompetenzen des Staates und dessen Mitwirkungskompetenzen bei den abgeleiteten Verbandskompetenzen der Europäischen Union miteinander verbindet. Das darin liegende Erfordernis, die Souveränitäts-, Herrschafts- und Legitimationsausprägung strukturell zu modifizieren, ist gerade aus der vom BVG aufgabengemäß eingenommenen Sicht der Selbstbehauptung der verfassten freiheitlichen und sozialstaatlichen Demokratie der Bundesrepublik Deutschland geboten, wenn der daraus gezogene Gemeinwohlgewinn im Sinne der Staatsziele der Bundesrepublik insgesamt höher ist. Diese Einschätzung vermag letztlich nur ein kluges politisches Gesamturteil vorzunehmen, das der spezifischen und konstruktiven Verantwortung 293 gerecht wird, die Deutschland als größtes Land in der Mitte des europäischen Kontinents dauerhaft für die europäische Integration trägt.

292 So zu Recht Norbert Röttgen. Vgl. Schmiese/Müller: CDU kritisiert Vorschläge der CSU, 2009.

293 Vgl. dazu das Vorwort zur 1. Auflage von Peter-Christian Müller-Graff: Vorwort, in: Peter-Christian MüllerGraff (Hrsg.): Deutschlands Rolle in der Europäischen Union, 1. Aufl., Baden-Baden 2008, S. 5. 